\title{
The imperfect fluid behind kinetic gravity braiding
}

\author{
Oriol Pujolàs, ${ }^{a}$ Ignacy Sawicki ${ }^{b}$ and Alexander Vikman ${ }^{c}$ \\ ${ }^{a}$ Departament de Física and IFAE, Universitat Autònoma de Barcelona, \\ 08193 Bellaterra, Barcelona, Spain \\ ${ }^{b}$ Institut für Theoretische Physik, Ruprecht-Karls-Universität Heidelberg, \\ Philosophenweg 16, 69120 Heidelberg, Germany \\ ${ }^{c}$ Theory Division, CERN, \\ CH-1211 Geneva 23, Switzerland \\ E-mail: pujolas@ifae.es, ignacy.sawicki@uni-heidelberg.de, \\ alexander.vikman@cern.ch
}

ABSTRACT: We present a standard hydrodynamical description for non-canonical scalar field theories with kinetic gravity braiding. In particular, this picture applies to the simplest galileons and k-essence. The fluid variables not only have a clear physical meaning but also drastically simplify the analysis of the system. The fluid carries charges corresponding to shifts in field space. This shift-charge current contains a spatial part responsible for diffusion of the charges. Moreover, in the incompressible limit, the equation of motion becomes the standard diffusion equation. The fluid is indeed imperfect because the energy flows neither along the field gradient nor along the shift current. The fluid has zero vorticity and is not dissipative: there is no entropy production, the energy-momentum is exactly conserved, the temperature vanishes and there is no shear viscosity. Still, in an expansion around a perfect fluid one can identify terms which correct the pressure in the manner of bulk viscosity. We close by formulating the non-trivial conditions for the thermodynamic equilibrium of this imperfect fluid.

Keywords: Cosmology of Theories beyond the SM, Classical Theories of Gravity, Global Symmetries, Nonperturbative Effects

ARXiv EPRINT: 1103.5360 


\section{Contents}

1 Introduction 1

2 Action and equations of motion $\quad 5$

3 Fluid picture $\quad 6$

$\begin{array}{lll}3.1 & \text { Four-velocity and kinematical decomposition } & 7\end{array}$

$\begin{array}{lll}3.2 & \text { Shift-charge or particle current } & 8\end{array}$

$\begin{array}{llr}3.3 & \text { Chemical potential and force } & 9\end{array}$

3.4 Energy-momentum tensor 11

3.5 Energy-momentum conservation and the first law of thermodynamics 12

$\begin{array}{ll}3.6 & \text { Equation of motion } \\ & 13\end{array}$

$\begin{array}{lll}3.7 & \text { Diffusion and non-dissipative bulk viscosity } & 15\end{array}$

$\begin{array}{llr}4 & \text { Equilibrium and vacuum configurations } & 19\end{array}$

5 How unnaturally complicated could it be? 20

6 Discussion and future directions $\quad 22$

$\begin{array}{ll}\text { A Choice of frames } & 24\end{array}$

B Action without second time derivatives $\quad 26$

\section{Introduction}

Scalar field theories with non-canonical derivative interactions, often referred to as $\mathrm{k}$-essence $[1,2]$, have gained significant attention in the past decade as novel and phenomenologically different models for the inflationary stage of the early universe $[3,4]$ and of the recent universe, dominated by dark energy ("DE"), see $[1,2,5]$ and dark matter ("DM"), see e.g. refs. [6-13]. The key new observable appearing in these theories in comparison to canonical scalar fields is the speed of sound which can be very different from the speed of light.

On the other hand, modifications of gravity like $[14,15]$ in the so-called decoupling limit $[16-18]^{1}$ reduce to non-canonical scalar field theory structurally different from k-essence. In particular, in Minkowski spacetime this scalar field theory by construction possesses the galilean symmetry: $\partial_{\mu} \phi \rightarrow \partial_{\mu} \phi+c_{\mu}$ where $c_{\mu}=$ const. A galilean-symmetric generalisation of this scalar field theory was introduced in $[21,22] .{ }^{2}$ Further, it was found

\footnotetext{
${ }^{1}$ For more recent development, see [19, 20]

${ }^{2}$ For earlier works unrelated to the decoupling limit, see e.g. [23-25]
} 
that it is impossible to covariantise these models in such a way so as to maintain the galilean symmetry for dynamical spacetimes in a self-consistent manner [26, 27]. Nonetheless, these theories have been named galileon theories and a significant body of work has already been carried out analysing their impact not only on cosmological solutions [28-45], but also in other circumstances [46-55]. In [56], we introduced a class of models which extends both $\mathrm{k}$-essence and the action of the DGP [14] decoupling limit by

$$
S_{\phi}=\int \mathrm{d}^{4} x \sqrt{-g}[K(\phi, \partial \phi)+G(\phi, \partial \phi) \square \phi]
$$

which we have utilised as an Imperfect Dark Energy (see also ref. [57], where this class of models was slightly later studied under the name $G$-inflation). ${ }^{3}$ This action does not possess the galilean symmetry and yet it does not contain a new degree of freedom, even in the presence of gravity. We have found a number of surprising features exhibited by this class of models. For example, contrary to k-essence and the ghost condensate [5], even in the limit where the scalar is an exact Goldstone boson, without direct couplings to matter, there exist attractors in expanding cosmologies in which the scalar field monitors and responds to the external energy density, only to eventually arrive at a final de-Sitter state; the null energy condition is generically violated in a stable manner and the system can evolve so as to cross the phantom divide. These features arise as a consequence of what we have named kinetic gravity braiding, the essential mixing of the derivatives of the scalar and of the metric, which cannot be undone by a field redefinition and which necessarily modifies gravity. The result of this kinetic braiding is that the energy-momentum tensor can no longer be brought to the perfect-fluid form. A number of works have followed up the analysis, investigating structure formation and inflation in this class of models [58-62].

In this work we extend our heretofore investigation of the cosmological solutions to generic backgrounds and analyse the models with kinetic gravity braiding as fluids. This turns out to be a very fruitful framework in which to proceed and we demonstrate that it results in a very significant simplification of the equations and in giving a very physical picture to the a priori complex dynamics of the system. For the complete list of fluid variables and notation see table 1.

We begin by recapping parts of our discussion on the equations of motion already presented in ref. [56] in section 2. Then, in section 3, we show that a fluid description is still possible. Just as in the case of k-essence, one identifies the time-like gradient of the scalar field with the fluid's velocity in this way choosing a local rest frame. This implies that the scalar field plays the role of the internal clock for the fluid. Since the shiftsymmetric Lagrangian is necessarily asymmetric with respect to $\phi \rightarrow-\phi$ there is a built-in arrow of time. Secondly one can realise that the equation of motion for the scalar takes the form of a divergence of a current, which is conserved in the case of a shift-symmetric Lagrangian. This allows us to identify the shift-charge density, $n$, in the local rest frame. What will prove key in the discussion here, is that this frame is neither the frame in which the charges are at rest, nor is it the frame in which there is no energy flux. However, it

\footnotetext{
${ }^{3}$ For a further generalisation of galileons and our action (1.1) see ref. [53]
} 
is in this particular frame that vorticity vanishes providing the hypersurface of constant intrinsic time on which the Cauchy data can be posed.

We follow Schutz [63] and interpret the derivative of the scalar field with respect to proper time as the chemical potential, $m$. The surprising new feature is that both the charge density and the the energy density now explicitly contain the expansion of the fluid elements. In addition to its thermodynamical part $P(m)$, the pressure, $\mathcal{P}$, contains a term proportional to the first time derivative of the chemical potential. As we will show, these terms are a signature of the fluid's generically being out of thermodynamical equilibrium. In addition, this fluid is now imperfect. Indeed, the energy flows neither along the field gradient nor along the shift current. ${ }^{4}$ Therefore the energy-momentum tensor contains offdiagonal energy-flow terms. This energy flow is, in fact, diffusion occurring along gradients of the chemical potential,

$$
\mathbf{q}=-\kappa \nabla m,
$$

where $\boldsymbol{\nabla}$ is the spatial gradient and $\kappa$ is the diffusivity. This is a dissipationless form of diffusion, with no entropy production. The fluid in this picture has zero temperature, is vorticity-free and does not have any shear viscosity, just one would expect of a system described by a single scalar field and an action. Thus it is natural to identify this fluid with an imperfect superfluid. The use of the fluid variables allows us to understand the dynamics described by the highly nonlinear Ampère-Monge-like equation of motion as the simple first law of thermodynamics

$$
\mathrm{d} E=-\mathcal{P} \mathrm{d} V+m \mathrm{~d} \mathcal{N},
$$

where $V$ is the comoving volume and $\mathcal{N}$ is the number of shift charges contained in $V$. In particular, the function $K$ in the Lagrangian (1.1) provides the equation of state for the fluid, while the function $G$ describes the dependence of the diffusivity on the chemical potential. Note that the usual Galileon choice for the function $G=(\partial \phi)^{2}$ is not necessarily one which is physically motivated in this fluid picture, as we discuss in section 5 .

In section 3.7, we show that when the fluid dynamics are expanded around the perfect fluid, with the diffusivity $\kappa$ employed as the expansion parameter, one obtains a correction to the pressure that is proportional to the expansion of the fluid elements, and therefore behaves in the manner of bulk viscosity. However, when a proper gradient expansion is taken, we show that this bulk viscosity disappears, confirming that there is no dissipation. That being said, the fluid does respond to the expansion, and therefore we propose that it is viscid in this generalised, non-dissipative sense. Secondly, in the incompressible limit, the equation of motion for the scalar field can be re-expressed as the diffusion equation for charge density

$$
\dot{n}=-\nabla(\mathfrak{D} \nabla n)+\ldots
$$

with the diffusion coefficient $\mathfrak{D} \equiv-\kappa(m \partial n / \partial m)^{-1}$, where we have ignored the terms arising from the non-inertial nature of the rest frame. Yet again we reiterate: this diffusion process is dissipationless and occurs at zero temperature.

\footnotetext{
${ }^{4}$ Note the this definition of imperfection is different from the one used in ref. [64, 65].
} 


\begin{tabular}{|llc|}
\hline Description & Notation & Definition \\
\hline Standard Kinetic Term & $X$ & $\frac{1}{2} g^{\mu \nu} \nabla_{\mu} \phi \nabla_{\nu} \phi$ \\
\hline Fluid Velocity & $u_{\mu}$ & $\nabla_{\mu} \phi / \sqrt{2 X}$ \\
\hline Chemical Potential & $m$ & $\sqrt{2 X}$ \\
\hline Expansion & $\theta$ & $\nabla_{\mu} u^{\mu}$ \\
\hline Spatial Projector & $\perp_{\mu \nu}$ & $g_{\mu \nu}-u_{\mu} u_{\nu}$ \\
\hline Time Derivative of $x$ & $\dot{x}$ & $u^{\mu} \nabla_{\mu} x$ \\
\hline
\end{tabular}

\begin{tabular}{|llrr|}
\hline Description & Notation & $k$-essence & Kinetic gravity braiding \\
\hline Lagrangian & $\mathcal{L}$ & $K(X)$ & $K(X)+G(X) \square \phi$ \\
\hline Diffusivity & $\kappa$ & 0 & $2 X G_{X}$ \\
\hline Thermal Pressure & $P$ & $K$ & $K$ \\
\hline Charge Density & $n$ & $\partial P / \partial m$ & $\partial P / \partial m+\kappa \theta$ \\
\hline Charge Current & $J_{\mu}$ & $n u_{\mu}$ & $n u_{\mu}-(\kappa / m) \perp_{\mu}^{\nu} \nabla_{\nu} m$ \\
\hline Total Pressure & $\mathcal{P}$ & $P$ & $P-\kappa \dot{m}$ \\
\hline Total Energy Density & $\mathcal{E}$ & $m n-P$ & $m n-P$ \\
\hline Energy Flow & $q_{\mu}$ & 0 & $q_{\mu}=-\kappa \perp_{\mu}^{\nu} \nabla_{\nu} m$ \\
\hline Diffusion Coefficient & $\mathfrak{D}$ & 0 & $-\kappa(m \partial n / \partial m)^{-1}$ \\
\hline Force Density & $f_{\mu}$ & $\perp_{\mu}^{\nu} \nabla_{\nu} P$ & $\perp_{\mu}^{\lambda} \nabla_{\lambda} P+\kappa \theta \perp_{\mu}^{\lambda} \nabla_{\lambda} m$ \\
\hline Energy Conservation & $u_{\nu} \nabla_{\mu} T^{\mu \nu}=0$ & $\mathrm{~d} E=-\mathcal{P} \mathrm{d} V$ & $\mathrm{~d} E=-\mathcal{P} \mathrm{d} V+m \mathrm{~d} \mathcal{N}$ \\
\hline
\end{tabular}

Table 1. Comparison of properties and notation of k-essence with the imperfect fluid of kinetic gravity braiding in the shift-symmetric case.

Using the fluid language for k-essence, we show that what is usually considered to be the relation between pressure and energy density (the "equation of state") is in fact nothing but the Euler relation for the thermodynamical representation of the fluid, relating the energy and pressure with the particle number and chemical potential. This relation guarantees the conservation of momentum. Surprisingly, the Euler relation is still valid for our fluid, but one must replace the total pressure with only its thermodynamical part.

In section 4, we turn to a discussion of the conditions required for thermodynamic equilibrium in our braided superfluid. A new result here (which also applies to k-essence, i.e. the usual perfect superfluid) is that, assuming our fluid interpretation, thermodynamic equilibrium implies the so-called Born-rigidity condition: the expansion and shear vanish; it turns out that the configuration must also be static. However, the key difference between 
these configurations and those considered previously (see e.g., [8]) is that the gradient of the scalar field remains time-like: therefore these type of equilibrium configurations only exist in theories with shift-symmetric Lagrangians. This also directly follows from our interpretation of $\phi$ as the intrinsic clock.

A new feature arising from kinetic gravity braiding is that the equation of motion for the scalar field provides a new condition which must be fulfilled by equilibrium configurations. In particular, this condition constrains the equation of state which the scalar must adopt. In the extreme case of a constant diffusivity $\kappa$, the total equation of state for the braided scalar together with external matter must be $w=-1 / 3$. In equilibrium, the scalar must arrange itself in such a way so as to screen the gravitational effects of itself and any external mass present in the system.

We close by describing the motion of degenerate relativistic fermions by a k-essence scalar-field theory in 5. While the former is a simple physical system, the k-essence describing it appears highly technically unnatural.

In table 1, we are providing a quick dictionary defining our terms and contrasting the hydrodynamical properties of k-essence and the fluid with kinetic gravity braiding. We assume the shift-symmetric case where the analogy with hydrodynamics is exact.

\section{Action and equations of motion}

In this section, we recap the discussion presented in our work [56, §2] where we introduced a class of scalar field theories minimally coupled to gravity, described by the action ${ }^{5}$

$$
S_{\phi}=\int \mathrm{d}^{4} x \sqrt{-g}[K+G \times B]
$$

where we have denoted

$$
X \equiv \frac{1}{2} g^{\mu \nu} \nabla_{\mu} \phi \nabla_{\nu} \phi, \text { and } B \equiv g^{\mu \nu} \nabla_{\mu} \nabla_{\nu} \phi \equiv \square \phi,
$$

and $K(\phi, X)$ and $G(\phi, X)$ are arbitrary functions of the scalar field and its standard kinetic term $X$. Here and in most of the paper, we use reduced Planck units where $M_{\mathrm{Pl}}=\left(8 \pi G_{\mathrm{N}}\right)^{-1 / 2}=1$. For further discussion, it is convenient to use the standard notation for the corresponding Lagrangian

$$
\mathcal{L}(\phi, X, B)=K(\phi, X)+G(\phi, X) \times B
$$

which we consider as a function of three independent variables $\phi, X$ and $B$. One should note that, if the system is symmetric with respect to constant shifts in field space: $\phi \rightarrow \phi+c$, then the Lagrangian is necessarily not symmetric with respect to $\phi \rightarrow-\phi$.

The Lagrangian $\mathcal{P}$ equivalent to $\mathcal{L}$ can be obtained by integrating by parts the scalarfield contribution $S_{\phi}$ of the action eq. (2.1):

$$
\mathcal{P}=K-\left[\left(\nabla^{\lambda} \phi\right) \nabla_{\lambda}\right] G=K-2 X G_{\phi}-G_{X} \nabla^{\lambda} \phi \nabla_{\lambda} X,
$$

\footnotetext{
${ }^{5}$ we use the metric signature convention $(+---)$.
} 
where the subscripts $\phi$ and $X$ denote partial differentiation with respect to these independent variables. It is clear that it is the dependence of $G$ on the field's gradient, $X$, that prevents this theory from being recast in a k-essence form, whereas the derivative of $G$ with respect to $\phi$ contributes as an additional k-essence term.

In ref. [56], we showed that the equation of motion for the scalar field takes the following form

$$
\nabla_{\mu} J^{\mu}=\mathcal{P}_{\phi},
$$

where $\mathcal{P}_{\phi}=K_{\phi}-\left[\left(\nabla^{\lambda} \phi\right) \nabla_{\lambda}\right] G_{\phi}$ and the current

$$
J_{\mu}=\left(\mathcal{L}_{X}-2 G_{\phi}\right) \nabla_{\mu} \phi-G_{X} \nabla_{\mu} X,
$$

corresponds to the Noether current for Lagrangians invariant under constant shifts in field space: $\phi \rightarrow \phi+c$. Thus this current is conserved provided that $\mathcal{P}_{\phi}=0$. The fully expanded equation of motion is somewhat unwieldy and was presented in ref. [56], see eq. (2.5) on page 6; it will not be necessary in the discussion here. We would like to stress that this second-order partial differential equation in the single scalar variable $\phi$ is the only dynamical equation besides the Einstein equations. In particular, this is the equation which one has to solve in numerical analysis. The presence of third-order derivatives might have been expected owing to the d'Alembertian term in the action; however, as we have proven in [56], the equation of motion contains at most second-order derivatives. Therefore theories with kinetic gravity braiding do not contain any additional hidden (and ghosty) degrees of freedom which would appear by virtue of the Ostrogradsky procedure. Further, for any coordinate $x^{i}$, the second derivative $\nabla_{i} \nabla_{i} \phi$ appears only linearly in the equation of motion. This allows one to associate this equation of motion with a particular generalisation of the Ampère-Monge equation for four-dimensional manifolds with Lorentzian signature. In section 3.6, we present a different derivation of the expanded equation of motion which makes use of the Raychaudhuri equation and explicitly shows how the higher-order derivatives are eliminated.

The energy-momentum tensor ("EMT") for the scalar field is most easily derived in the standard way using the Lagrangian presented in eq. (2.3)

$$
T_{\mu \nu} \equiv \frac{2}{\sqrt{-g}} \frac{\delta S_{\phi}}{\delta g^{\mu \nu}}=\mathcal{L}_{X} \nabla_{\mu} \phi \nabla_{\nu} \phi-g_{\mu \nu} \mathcal{P}-\nabla_{\mu} G \nabla_{\nu} \phi-\nabla_{\nu} G \nabla_{\mu} \phi
$$

It is key to observe that the EMT also contains second derivatives of $\phi$ appearing in $\mathcal{P}, \mathcal{L}_{X}$ and $\nabla_{\mu} G$. Therefore as we have already discussed in ref. [56], both the Einstein's equations as well as the equation of motion for the scalar field (2.4) contain second derivatives of both the metric $g_{\mu \nu}$ and the scalar field $\phi$, so that the system is not diagonal in the second derivatives and no field redefinition exists which would unmix the two fields. It is this essential kinetic mixing of gravity and the scalar that we have named kinetic gravity braiding.

\section{Fluid picture}

When considering scalar-field theories as applied to cosmology, one usually restricts to the case with timelike field derivatives. It is well known that many aspects of k-essence 
in such a setup can be described in terms of relativistic hydrodynamics [3]. Below we introduce a fluid picture for the theory given by the action (2.1). As in the case of kessence, this hydrodynamical/fluid language turns out to be helpful in developing intuition and in simplifying notation. To our best knowledge the analysis presented below appears in the literature for the first time. For earlier studies of thermodynamics of relativistic potential flows and k-essence see e.g. refs. [66-69].

\subsection{Four-velocity and kinematical decomposition}

First of all, by analogy with k-essence we can introduce an effective four-velocity

$$
u_{\mu} \equiv \frac{\nabla_{\mu} \phi}{\sqrt{2 X}}
$$

which defines the local rest frame (LRF). We will then use the notation

$$
(\dot{)})=\frac{\mathrm{d}}{\mathrm{d} \tau}()=u^{\alpha} \nabla_{\alpha}(),
$$

for the derivative along $u^{\alpha}$ (material derivative), thus making $\tau$ the proper time of an observer comoving with the LRF. We can then introduce the transverse projector

$$
\perp_{\mu \nu}=g_{\mu \nu}-u_{\mu} u_{\nu}
$$

which will be the first fundamental form in the hypersurfaces $\Sigma_{\phi}: \phi(x)=$ const and which will allow us to decompose vectors (and gradients) into time-like and space-like parts as observed in the LRF,

$$
\nabla_{\mu}=u_{\mu} u^{\lambda} \nabla_{\lambda}+\perp_{\mu}^{\lambda} \nabla_{\lambda}=u_{\mu} \frac{\mathrm{d}}{\mathrm{d} \tau}+\bar{\nabla}_{\mu}
$$

Without loss of generality, we will assume that the time derivative of $\phi$ is positive definite, always making $u^{\mu}$ future directed. The field $\phi$ can thus be considered to be an internal clock. The hypersurfaces $\Sigma_{\phi}$ then are Cauchy hypersurfaces for the standard-model fields and gravity and natural candidates for being the Cauchy hypersurfaces for the equation of motion of the field $\phi$ (see the discussion regarding this particular choice of frame in appendix A). The equation of motion (2.4) is of the second order, therefore from the Cauchy-Kowalewski theorem, the initial data on $\Sigma_{\phi}$ are $\dot{\phi}_{0}(\boldsymbol{x})$ and $\phi_{0}(\boldsymbol{x})$, which is represented not only by its value $\left(\phi_{0}(\boldsymbol{x})=\right.$ const on $\left.\Sigma_{\phi}\right)$ but also by second derivatives with respect to the spatial coordinates $\boldsymbol{x}$ on $\Sigma_{\phi}$.

In the shift-symmetric case, the Lagrangian is explicitly independent of the internal clock $\phi$. Then, as we have already mentioned, the Lagrangian necessarily violates $\phi \rightarrow-\phi$ symmetry furnishing our model with a built-in arrow of time or an asymmetry of the motions with respect to an increasing or decreasing internal clock $\phi$.

The corresponding four-acceleration is defined as

$$
a_{\mu} \equiv \dot{u}_{\mu} \equiv u^{\lambda} \nabla_{\lambda} u_{\mu}
$$


and can also be rewritten in form of a spatial gradient,

$$
a_{\mu}=\perp_{\mu}^{\lambda} \nabla_{\lambda} \ln \dot{\phi}
$$

It will be convenient to make use of the notation for its magnitude,

$$
a \equiv \sqrt{-a^{\lambda} a_{\lambda}} .
$$

Further, we can make the standard kinematical decomposition,

$$
\nabla_{\mu} u_{\nu}=u_{\mu} a_{\nu}+\frac{1}{3} \theta \perp_{\mu \nu}+\sigma_{\mu \nu},
$$

where $\theta$ denotes the expansion,

$$
\theta \equiv \perp_{\mu}^{\lambda} \nabla_{\lambda} u^{\mu}=\nabla_{\mu} u^{\mu}
$$

and $\sigma_{\mu \nu}$ is the shear tensor,

$$
\sigma_{\mu \nu} \equiv \frac{1}{2}\left(\perp_{\mu}^{\lambda} \nabla_{\lambda} u_{\nu}+\perp_{\nu}^{\lambda} \nabla_{\lambda} u_{\mu}\right)-\frac{1}{3} \perp_{\mu \nu} \theta,
$$

which is symmetric, traceless and purely spatial. In this decomposition, we have used the vanishing of the rotation tensor (or twist) $\perp_{\mu}^{\lambda} \nabla_{\lambda} u_{\nu}-\perp_{\nu}^{\lambda} \nabla_{\lambda} u_{\mu}$ in accordance with the Frobenius theorem. The four-acceleration $a_{\mu}$, expansion $\theta$ and the shear tensor $\sigma_{\mu \nu}$ are constructed from spatial derivatives of the scalar field and can be calculated on any $\Sigma_{\phi}$ directly from the initial data without using the equation of motion. Finally, it is useful to introduce the extrinsic curvature of the hypersurface $\Sigma_{\phi}$,

$$
\mathcal{K}_{\alpha \beta}=\perp_{\alpha}^{\gamma} \perp_{\beta}^{\lambda} \nabla_{\lambda} u_{\gamma}=\sigma_{\alpha \beta}+\frac{1}{3} \perp_{\alpha \beta} \theta
$$

from whence it follows that $\mathcal{K}=\theta$.

\subsection{Shift-charge or particle current}

Using the formulae

$$
\nabla_{\mu} X=2 X a_{\mu}+\dot{X} u_{\mu}, \text { and } \square \phi=\frac{\dot{X}}{\sqrt{2 X}}+\sqrt{2 X} \theta,
$$

we can write the Noether current eq. (2.5), conserved only when shift symmetry is present, as

$$
J_{\mu}=\left(\dot{\phi}\left(K_{X}-2 G_{\phi}\right)+\kappa \theta\right) u_{\mu}-\kappa a_{\mu},
$$

where we have introduced the notation ${ }^{6}$

$$
\kappa \equiv 2 X G_{X} .
$$

\footnotetext{
${ }^{6}$ Note that in ref. [56, Eq. (3.6)], we used a definition for $\kappa$ that is smaller than this one by a factor of $2, \kappa_{\text {here }}=2 \kappa_{\text {there }}$. This served to simplify the equations even more in the particular case of a cosmological background, but the proper normalisation for this term which gives its physical meaning is the one presented in this work.
} 
From the standard discussion of imperfect fluids (see e.g. ref. [70]), we have to identify the space-like part of the current $(3.13),-\kappa a_{\mu}$, with diffusion, while $\kappa$ can be interpreted as the diffusivity, which we will take to be the coefficient relating the energy flow to the gradient of the chemical potential ${ }^{7}$ (see eq. (3.35)). Despite the presence of second derivatives in (2.5), the current does not contain the second time derivative of the field $\phi$ and can be calculated directly from initial data. The density of the shift-symmetry charge ${ }^{8}$

$$
n \equiv u^{\mu} J_{\mu}=\dot{\phi}\left(K_{X}-2 G_{\phi}\right)+\kappa \theta,
$$

contains the expansion $\theta$, which is highly non-standard. It is convenient to associate this Noether current with the particle-number current. In such a way, the introduced total particle/charge number will be conserved provided there be shift symmetry. However, the number of particles $\mathcal{N} \equiv n V$ in an infinitesimal volume $V$ moving with velocity $u^{\mu}$ (comoving volume) is not conserved. Indeed using the decomposition (3.13) one can write equation of motion (2.4) in the form

$$
\dot{n}+\theta n-\nabla_{\mu}\left(\kappa a^{\mu}\right)=\mathcal{P}_{\phi}
$$

and using the geometric meaning of the expansion

$$
\theta=\frac{\dot{V}}{V}
$$

we can obtain

$$
\dot{\mathcal{N}}=V\left(\mathcal{P}_{\phi}+\nabla_{\mu}\left(\kappa a^{\mu}\right)\right)=V \mathcal{P}_{\phi}+\dot{\mathcal{N}}_{\text {dif }}
$$

where $\dot{\mathcal{N}}_{\text {dif }}$ denotes the number of particles transported to the volume by diffusion per unit of proper time. Thus generically $\mathcal{N}$ is not conserved even in the shift-symmetric case. The total particle current is subluminal provided

$$
n^{2}-\kappa^{2} a^{2} \geq 0
$$

If eq. (3.19) holds, then one can chose an alternative fluid description where one takes the Eckart frame which moves with the particles as the LRF. We will comment more on this later.

\subsection{Chemical potential and force}

Using the general expression for the Energy-Momentum Tensor ("EMT") (2.6) and the decomposition (3.8), we define the energy density of the fluid in the standard way

$$
\mathcal{E} \equiv T_{\mu \nu} u^{\mu} u^{\nu}=2 X\left(K_{X}-G_{\phi}\right)+\kappa \theta \dot{\phi}-K .
$$

\footnotetext{
${ }^{7}$ Note that usually the terms diffusivity and diffusion coefficient are used interchangeably, since the same coefficient usually appears in both the Fick's laws. We have kept the definition of diffusivity as the one here and use it to mean a coefficient which determines an energy flux rather than a charge flux: in our case, this is a more natural definition. In section 3.7 we show that, in an expansion around an incompressible fluid, the equation of motion reduces to the diffusion equation with the diffusion coefficient related to, but not the same as, the diffusivity, see eq. (3.59).

${ }^{8}$ Note that in ref. [56, Eq. (3.2)], we denoted this number density by $J: n_{\text {here }}=J_{\text {there }}$.
} 
Contrary to the usual descriptions of relativistic imperfect fluids, the energy density contains the expansion, $\theta$.

For the shift-symmetric case we recall the definition of the effective mass per charge (which is also the chemical potential or injection energy) see e.g. [71, p. 562] and obtain

$$
m \equiv\left(\frac{\partial \mathcal{E}}{\partial n}\right)_{\tau, V=\text { const }}=\left(\frac{\partial \mathcal{E}}{\partial n}\right)_{\phi=\text { const }, \theta=0}=\dot{\phi}
$$

this expression is identical to that of ref. [63]. Further, we will identify

$$
m \equiv \dot{\phi}=\sqrt{2 X}>0
$$

with an effective rest mass per particle building the fluid even in non-shift-symmetric cases. As we will see later (see eqs.(3.37), (3.41)) the rest-mass introduced in this way plays the role of the chemical potential. ${ }^{9}$

The effective momentum of the particle can then be defined as

$$
p_{\mu} \equiv m u_{\mu}
$$

and the corresponding relativistic vorticity, $\nabla_{\mu} p_{\nu}-\nabla_{\nu} p_{\mu}$, is zero. ${ }^{10}$ Further, the fact that $p_{\mu} p^{\mu}=m^{2}$ and $p_{\mu}=\nabla_{\mu} \phi$, means that $-\phi$ plays the role of Hamilton's principal function (action as a function of final coordinates) for each shift-charge. Indeed, for the action of a particle we have

$$
s=-\int m d \tau=-\phi,
$$

where we have used eq. (3.22) and where we have omitted the constant of integration.

Exploiting the particle momentum, one can introduce a relativistic 3-force $F_{\mu}$ acting on each particle,

$$
F_{\mu} \equiv \perp_{\mu}^{\lambda} \dot{p}_{\lambda}=\perp_{\mu}^{\lambda} \frac{\mathrm{d}}{\mathrm{d} \tau}\left(m u_{\lambda}\right)
$$

Then, using the decomposition

$$
\nabla_{\mu} m=m a_{\mu}+\dot{m} u_{\mu}
$$

which follows from the definition of the effective mass (3.22), and decomposition (3.8), the force can be written as the gradient of the chemical potential:

$$
F_{\mu}=m a_{\mu}=\perp_{\mu}^{\lambda} \nabla_{\lambda} m .
$$

Here it is worth remembering that, in a system in thermodynamical equilibrium which experiences an external potential, the chemical potential is given by this external potential

\footnotetext{
${ }^{9}$ In the literature, $m$ is also called specific enthalpy or Gibbs energy per particle.

${ }^{10}$ Here it is worth recalling that in a stationary gravitational field with Killing vector $\partial_{t}$ the conserved energy of a particle is governed by $p_{t}$. Using our identification we obtain that $p_{t}=\partial_{t} \phi$ has to be conserved. As it was shown for k-essence in [72], the energy $p_{t}$ given by (3.23) is indeed conserved for stationary configurations. Taking into account that $\phi$ plays the role of an internal clock one can expect that stationarity implies shift symmetry in field space. Thus we conjecture that the statement from [72] is also applicable to the kinetic gravity braiding theories.
} 
plus some constant, so that the force acting on the particle is exactly the gradient of the external potential, as expected (do not forget the signature). We discuss equilibrium configurations in detail in section 4 .

Further we can also express the current eq. (3.13) making use of the chemical potential

$$
J_{\mu}=n u_{\mu}-\frac{\kappa}{m} \perp_{\mu}^{\lambda} \nabla_{\lambda} m,
$$

c.f. with the formula (59.5) for the diffusive current from ref. [66, p. 231].

\subsection{Energy-momentum tensor}

In this fluid language the EMT eq. (2.6) can be re-expressed as

$$
T_{\mu \nu}=\mathcal{E} u_{\mu} u_{\nu}-\perp_{\mu \nu} \mathcal{P}+u_{\mu} q_{\nu}+u_{\nu} q_{\mu},
$$

where the energy density is

$$
\mathcal{E} \equiv T_{\mu \nu} u^{\mu} u^{\nu}=2 X\left(K_{X}-G_{\phi}\right)+\theta m \kappa-K .
$$

As we have already remarked, contrary to the usual descriptions of relativistic imperfect fluids, the energy density contains the expansion, $\theta$. Still, this expression can be calculated purely from the initial data.

Further, we can define the effective total isotropic pressure in the usual fashion and find that it is given by the Lagrangian eq. (2.3):

$$
\mathcal{P} \equiv-\frac{1}{3} T^{\mu \nu} \perp_{\mu \nu}=K-2 X G_{\phi}-\kappa \dot{m} .
$$

The presence of the last term containing $\dot{m}$ can be associated with an additional force transporting particles along the gradients of the chemical potential. Because of this term, the total pressure can be calculated only after making use of the equations of motion which is not the case in standard fluids (see section 3.6 for a further discussion). However, in configurations in thermodynamical equilibrium this term vanishes. It will prove helpful to also introduce the part of pressure

$$
P \equiv K-2 X G_{\phi}
$$

which can be directly calculated from the initial data and which does not vanish in equilibrium configurations, usually called the thermodynamic pressure. ${ }^{11}$

Finally the energy flow associated with diffusion is

$$
q_{\mu} \equiv \perp_{\mu \lambda} T_{\nu}^{\lambda} u^{\nu}=-m \kappa a_{\mu}=m \perp_{\mu \nu} J^{\nu} .
$$

Thus the energy flow $q_{\mu}$ arises purely from diffusive part of the particle current $\perp_{\mu \nu} J^{\nu}$ and the heat flow, which is defined as ${ }^{12}$

$$
\text { Heat Flow } \equiv \perp_{\mu \lambda} T_{\nu}^{\lambda} u^{\nu}-m \perp_{\mu \nu} J^{\nu}=0,
$$

\footnotetext{
${ }^{11}$ This imperfection from the construction called Inhomogeneous Equation of State where pressure is postulated to be a function of both the energy density and the Hubble parameter, see e.g. [73-75].

${ }^{12}$ See e.g. ref. [76, $§ 1$, eq. (39)]. Note that many works use the Eckart frame as the LRF from the very beginning so that the $q_{\mu}$ used there can only be the heat flow.
} 
vanishes, as it should for a system with identically zero temperature. Then we can see that the diffusive energy flux is proportional to the spatial gradient of the chemical potential:

$$
q_{\mu}=-\kappa \perp_{\mu}^{\nu} \nabla_{\nu} m
$$

c.f. with the formula (59.5) for the diffusive energy flux from [66, p. 231]. We take this relation as our definition of $\kappa$ as the diffusivity. It is instructive to compare these relations with formulae (285) and (287) from the imperfect fluid from review [70, p. 58].

In simple equilibrium thermodynamics, the chemical potential has to be equal throughout the body. If there are gradients of the chemical potential, then the system develops forces and transports particles to compensate for these gradients. As we discuss in section 4 , in our case it is possible to construct equilibrium configurations which do nonetheless exhibit diffusive flow in a time-invariant fashion.

Having defined the thermodynamic quantities, we can then re-express the particle density eq. (3.15)

$$
n(\phi, m, \theta)=P_{m}+\kappa \theta+m \kappa_{\phi}=\mathcal{P}_{m}+\nabla_{\mu}\left(\kappa u^{\mu}\right),
$$

where we have explicitly stressed that we are treating the expansion as an independent variable. This form allows us to immediately see that $n$ can be found using solely initial data.

Using equations (3.15), (3.22), (3.30) and (3.32) we can obtain the Euler relation:

$$
\mathcal{E}=m n-P .
$$

Observe that the diffusion contribution $\kappa \dot{m}$ cancels out and only the usual equilibrium part of the pressure, which can be calculated from the initial data, contributes. We should stress here that all the above results hold even when shift-symmetry is broken and particle number is not conserved.

We would like to mention that the result eq. (3.37) in the k-essence limit $\left(G_{X}=0\right)$ reduces to the formula sometimes referred to as the k-essence equation of state, $\mathcal{E}=$ $2 X P_{X}-P[1]$. To our best knowledge, the relationship between this equation of state and the Euler relation appears to have been missed in the literature.

\subsection{Energy-momentum conservation and the first law of thermodynamics}

It is instructive to analyse the equations resulting from the conservation of the EMT $\nabla_{\mu} T_{\nu}^{\mu}=0$. For energy conservation we obtain:

$$
u_{\nu} \nabla_{\mu} T^{\mu \nu}=\dot{\mathcal{E}}+\theta(\mathcal{E}+\mathcal{P})-\nabla_{\lambda}\left(m \kappa a^{\lambda}\right)+m \kappa a_{\lambda} a^{\lambda}=0
$$

where we have used the definition of the expansion and the property of four acceleration $u_{\mu} a^{\mu}=0$. Further we can express the divergence of the diffusion current through the equation of motion (3.16)

$$
\nabla_{\mu}\left(\kappa a^{\mu}\right)=m\left(\dot{n}+\theta n-\mathcal{P}_{\phi}\right)
$$


so that the conservation of energy eventually reduces to

$$
\dot{\mathcal{E}}+\theta(\mathcal{E}+\mathcal{P})-m(\dot{n}+\theta n)+m \mathcal{P}_{\phi}=0 .
$$

Let us now consider the evolution of the energy $E \equiv \mathcal{E} V$ in the comoving volume $V$. Recalling the definition of the expansion (3.17) and the "particle conservation" equation (3.18) we obtain the first law of thermodynamics for the matter in the comoving volume in the shift-symmetric case:

$$
\mathrm{d} E=-\mathcal{P} \mathrm{d} V+m \mathrm{~d} \mathcal{N}
$$

where $\mathcal{N}$ is number of particles in the volume $V$, while d denotes the differential along the velocity $u^{\mu}$. From expression (3.41) it is obvious that $m$ plays the role of chemical potential. In the general case with $\mathcal{P}_{\phi} \neq 0$, one should modify eq. (3.41) by substituting d $\mathcal{N} \rightarrow \delta \mathcal{N}_{\text {dif }}$ - the infinitesimal number of particles transported to the volume by diffusion, but not those produced by the source $\mathcal{P}_{\phi}$.

There is only one single scalar degree of freedom $\phi$ and one independent scalar equation of motion (2.4). Hence $\perp_{\mu \nu} \nabla_{\lambda} T^{\lambda \nu}=0$, momentum conservation, cannot contain any additional information. Indeed, one can calculate that

$$
\perp_{\mu \nu} \nabla_{\lambda} T^{\lambda \nu}=(\mathcal{E}+P-m n) a_{\mu},
$$

therefore the Euler relation (3.37) guarantees momentum conservation.

For completeness, it is worth mentioning how the "Archimedes law" changes for this fluid. The force density is given by

$$
f_{\mu} \equiv n F_{\mu},
$$

or applying the Euler relation (3.37) it can be written as

$$
f_{\mu}=n \perp_{\mu}^{\lambda} \nabla_{\lambda} m=n m a_{\mu}=(\mathcal{E}+P) a_{\mu} .
$$

On the other hand, using momentum conservation $\perp_{\mu \nu} \nabla_{\lambda} T^{\lambda \nu}=0$, after some algebra we obtain

$$
f_{\mu}=\perp_{\mu}^{\lambda} \nabla_{\lambda} P+\left(\kappa_{\phi} m+\kappa \theta\right) \perp_{\mu}^{\lambda} \nabla_{\lambda} m .
$$

Hence the force density originates from a superposition of spatial gradients of the equilibrium part of pressure and of the chemical potential. Using eq. (3.36), we can rewrite the contents of the parentheses as $\kappa_{\phi} m+\kappa \theta=n-P_{m}$, which disappears in thermodynamical equilibrium (it is one of the Maxwell relations). On the other hand, in k-essence, the last term is not present by the simple virtue of $\kappa=0$.

\subsection{Equation of motion}

Let us discuss the equation of motion (3.16) in more detail. In particular, we will be interested in solving it with respect to highest time derivative: $\dot{m}=\ddot{\phi}$. Naively it seems that eq. (3.16) should have third derivatives. However, as we know from ref. [56] these terms 
with third derivatives cancel. Indeed, using eq. (3.26), (3.31) and the Euler integral (3.37) we obtain for eq. (3.16)

$$
\left(n_{m}+\kappa_{\phi}\right) \dot{m}+\kappa\left(\dot{\theta}-\nabla_{\mu} a^{\mu}\right)+\theta n-m \kappa_{m} a^{\lambda} a_{\lambda}+\mathcal{E}_{\phi}=0,
$$

while from the Raychaudhuri equation we have

$$
\dot{\theta}=-\frac{1}{3} \theta^{2}+\nabla_{\mu} a^{\mu}-\sigma_{\mu \nu} \sigma^{\mu \nu}-R_{\mu \nu} u^{\mu} u^{\nu},
$$

so that third derivatives disappear giving a second-order equation

$$
\left(n_{m}+\kappa_{\phi}\right) \dot{m}-\kappa\left(\sigma_{\alpha \beta} \sigma^{\alpha \beta}+\frac{1}{3} \theta^{2}+R_{\mu \nu} u^{\mu} u^{\nu}\right)+\theta n-\kappa_{m} m a^{\lambda} a_{\lambda}+\mathcal{E}_{\phi}=0 .
$$

We then use the Gauss equation

$$
\sigma_{\alpha \beta} \sigma^{\alpha \beta}-\frac{2}{3} \theta^{2}=-{ }^{(3)} R-2 G_{\mu \nu} u^{\mu} u^{\nu}
$$

to re-express the shear in terms of the intrinsic curvature of the spatial hypersurface and obtain for the equation of motion (3.48)

$$
\left(n_{m}+\kappa_{\phi}\right) \dot{m}+\theta n+\kappa\left({ }^{(3)} R+2 G_{\mu \nu} u^{\mu} u^{\nu}-R_{\mu \nu} u^{\mu} u^{\nu}-\theta^{2}\right)-\kappa_{m} m a^{\lambda} a_{\lambda}+\mathcal{E}_{\phi}=0 .
$$

This equation of motion is linear in the highest time derivative $\ddot{\phi}=\dot{m}$. Using Einstein equations and the expression for the EMT (3.29), we can eliminate the curvature term

$$
2 G_{\mu \nu} u^{\mu} u^{\nu}-R_{\mu \nu} u^{\mu} u^{\nu}=\frac{3}{2}(\mathcal{E}-P+\kappa \dot{m})+W_{\text {ext }},
$$

where we have denoted any possible contribution to the above contraction of the curvature tensors external to the scalar field as

$$
W_{\text {ext }} \equiv\left(T_{\text {ext }}^{\mu \nu}+\frac{1}{2} T_{\text {ext }} g^{\mu \nu}\right) u_{\mu} u_{\nu}
$$

Thus after such an elimination of the second derivatives of the metric, the equation of motion takes the form

$$
\left(n_{m}+\kappa_{\phi}+\frac{3}{2} \kappa^{2}\right) \dot{m}+\theta n+\kappa\left({ }^{(3)} R+\frac{3}{2}(\mathcal{E}-P)-\theta^{2}\right)-\kappa_{m} m a^{\mu} a_{\mu}+\mathcal{E}_{\phi}=-\kappa W_{\mathrm{ext}} .
$$

The diffusivity $\kappa$ also serves as an effective coupling constant in front of the external source term $W_{\text {ext }}$. Since the total pressure $\mathcal{P}$ contains $\dot{m}$ (see eq. (3.31)), the above equation implies that the pressure of the fluid depends on the acceleration and the extrinsic curvature tensor (and therefore the expansion $\theta$ ). Moreover, we should expect that the coefficient in front of $\ddot{\phi}$ is responsible for the presence or absence of ghosts / negative kinetic energies. Namely, there are no ghosts provided

$$
D \equiv n_{m}+\kappa_{\phi}+\frac{3}{2} \kappa^{2}>0 .
$$


We confirm this claim explicitly in our discussion of the effective acoustic metric for perturbations in the follow-up work ref. [77]. As we have already mentioned, this condition is not correlated with the Null Energy Condition. Using eq. (3.37) and eq. (3.36) this function $D$ can also be written in the form

$$
D=\frac{\mathcal{E}_{m}-\kappa \theta}{m}+\frac{3}{2} \kappa^{2} .
$$

which simply reduces to the well-known k-essence limit, $D=\mathcal{E}_{X}$ [78]. Provided $D \neq 0$, the solution of the Cauchy problem exists locally under the usual limitations. On the other hand, from the equation of motion (3.53) it follows that the zeroes of $D$ generically correspond to pole-like singularities in $\dot{m}$. Given the dependence of the total pressure on $\dot{m}$, eq. (3.31), the configurations with $D=0$ are pressure singularities and they build a barrier in phase space which is generically impenetrable. Thus a fluid evolving from some initial data with healthy perturbations $(D>0)$ will never become ghosty in a smooth and controllable way. Note that, similarly to the cases studied in ref. [78], for some Lagrangians and some external matter $W_{\text {ext }}$ there may well exist trajectories penetrating this barrier. However, these trajectories would have measure zero in the phase space of the system since they correspond to an exact cancellation of the above singularity. Finally, we would like to stress that the fluid variables are helpful to develop intuition but do not appear to be very practical in actual numerical calculations. The equation of motion cannot be reduced to system of ordinary differential equations along a world line. In particular, the system (3.53) and (3.47) is not closed and requires quite the unwieldy Raychaudhuri equation for the shear tensor $\sigma_{\mu \nu}$ which has to be supplemented with an even more complicated equation for $\dot{a}_{\mu}$. On top of that all these equations will be subject to the constraint that the vorticity of $u^{\mu}$ vanish. Working with $\phi$ directly and solving the expanded (2.4) has the advantage that there are no further constraints. Solving equations of motion for the velocity potential (phase for superfluids) $\phi$ is of course the standard practice in vorticity-free hydrodynamics.

\subsection{Diffusion and non-dissipative bulk viscosity}

Let us now elaborate on the diffusive interpretation of this fluid. First of all, one quickly notices that eq. (3.28) is a "1st Fick's law" $J \propto \bar{\nabla} m$, relating the diffusive flux of particles in or out of a comoving volume to the spatial gradient of the chemical potential, which again is consistent with our interpretation of $\kappa$ as the diffusivity. Of course, the "2nd Fick's law", namely a diffusion-type equation for the charge density $n$, can be identified with the equation of motion. Turning the attention to the shift-symmetric case for simplicity, we can rewrite the equation of motion (3.39) as

$$
\dot{n}+\theta n=n_{m} \dot{m}+\theta n+\kappa \dot{\theta}=\nabla_{\mu}\left(\frac{\kappa}{m} \perp^{\mu \nu} \nabla_{\nu} m\right),
$$

which is already a time-dependent diffusion-type equation for $m$, with some extra terms. As we have already mentioned, the $\kappa \dot{\theta}$ term plays a crucial role to keep this equation of second order, through the Raychaudhury equation. One could worry that at the same time this may remove the diffusive character of eq. (3.56), but this is actually not the case. For 
instance, there are configurations in which one can have a nonzero $\nabla_{\mu} a^{\mu}$ but still $\dot{\theta}=0$ (in section 4 we discuss examples of such configurations).

It is perhaps more illuminating to write eq. (3.56) directly in terms of $n$. For this purpose, it suffices to realise that since $n=n(m, \theta)$ then at least locally one can express $m=m(n, \theta)$. Performing a gradient expansion (i.e., in the expansion in $\kappa$ ) one then has $\nabla_{\mu} m=\frac{1}{n_{m}} \nabla_{\mu} n+O(\kappa)$ with $n$ and $n_{m}$ taken at $\theta=0$, that is for the perfect fluid limit. With this, the equation of motion (or charge conservation) (3.16) reduces to

$$
\dot{n}+\theta n=\nabla_{\lambda}\left(\frac{\kappa}{n_{m} m} \perp^{\mu \nu} \nabla_{\nu} n\right)+O\left(\kappa^{2}\right) .
$$

Bar the terms involving the expansion, this equation is the standard diffusion equation

$$
\dot{n}=-\bar{\nabla}_{\mu}\left(\mathfrak{D} \bar{\nabla}^{\mu} n\right)+\mathfrak{D} a^{\mu} \bar{\nabla}_{\mu} n,
$$

with the diffusion coefficient a function of the diffusivity ${ }^{13}$

$$
\mathfrak{D}=-\frac{\kappa}{n_{m} m}=-\frac{\kappa}{n} c_{\mathrm{s}}^{2} \equiv-\tau_{\mathfrak{D}} c_{\mathrm{s}}^{2},
$$

with $\tau_{\mathfrak{D}}$ the diffusion timescale appearing here since the diffusion coefficient has dimensions of $\mathrm{L}^{2} / \mathrm{T}$. In the limit of an incompressible fluid, eq. (3.58) is in fact the exact equation of motion to all orders in $\kappa$. The last term in this diffusion equation is proportional to acceleration and manifests that the equation is written in a non-inertial accelerated frame. Note that in this discussion we have not used the Einstein equations. The whole procedure is based on the presence of a higher derivative in the equation of motion, and the corresponding $\nabla_{\mu} a^{\mu}$ in the equation of motion i.e. charge conservation (3.16).

One may wonder whether the system gives enough freedom to ever realise this diffusive behaviour. It can be seen that this can occur when the initial data are such that the expansion $\theta$ vanishes and $\dot{\theta}$ is tuned to be small. This means that the timescale for the expansion of the volume is much larger than the timescale for the diffusion,

$$
\tau_{\mathfrak{D}} \ll \theta^{-1} .
$$

This condition also happens to imply that the contribution from the expansion to the particle number is negligible. Then we must require that, in the charge-conservation equation (3.16), the expansion term is negligible compared to divergence term, or roughly

$$
\theta n \ll \kappa \nabla_{\mu} a^{\mu} \quad \Rightarrow \quad \nabla_{\mu} a^{\mu} \gg \tau_{\mathfrak{D}}^{-2} .
$$

Finally, one needs to make sure that the configuration is maintained for sufficient time, i.e. that $\theta$ evolves slowly enough. Through the Raychaudhuri equation (3.47) we require that

$$
\begin{aligned}
& \frac{\kappa}{n}\left|\nabla_{\mu} a^{\mu}\right| \gg \dot{\theta} \tau_{\mathfrak{D}} \approx\left|\nabla_{\mu} a^{\mu}\right| \tau_{\mathfrak{D}}-\sigma_{\alpha \beta} \sigma^{\alpha \beta} \tau_{\mathfrak{D}} \\
& \Rightarrow \nabla_{\mu} a^{\mu} \gg\left|\nabla_{\mu} a^{\mu}-\sigma_{\alpha \beta} \sigma^{\alpha \beta}\right|
\end{aligned}
$$

\footnotetext{
${ }^{13}$ It is useful to compare this expression for $\mathfrak{D}$ with [66, Eq. (59.9), p. 232]. Note that our $\kappa / m=-\alpha$ and $\rho c=n$.
} 
where all the above quantities depend purely on initial data. The final condition is precisely the tuning that we are required to perform on these initial conditions to ensure that diffusion occurs for some finite time much longer than the characteristic diffusion timescale $\tau_{\mathfrak{D}}$. This is a rather fine-tuned situation, which is already clear from the fact that this cancellation is not possible in $1+1$ dimensions. However, initial conditions obeying (3.62) definitely exist. For example, in $1+3$ dimensional Minkowski spacetime, these initial conditions can be easily realised by the following ansatz in Cartesian coordinates $(t, \mathbf{x}): \partial_{t} \phi_{0}(\mathbf{x})=1$ and $\phi_{0}(\mathbf{x})=\epsilon f(\mathbf{x})$, where $f(\mathbf{x})$ is a harmonic function, $\Delta f(\mathbf{x})=0$, and $\epsilon$ is a small parameter. In that case the geometric invariants are $\theta=\mathcal{O}\left(\epsilon^{3}\right), \nabla_{\mu} a^{\mu}=\mathcal{O}\left(\epsilon^{2}\right)$ and $\sigma^{\mu \nu} \sigma_{\mu \nu}=\mathcal{O}\left(\epsilon^{2}\right)$, whereas from the Raychaudhuri equation we get $\dot{\theta}=\mathcal{O}\left(\epsilon^{4}\right)$.

We conclude that the interpretation of this system as a diffusive one seems appropriate and robust. We emphasise that this is a non-dissipative form of diffusion present at zero temperature. Further one can see that $\mathfrak{D} a^{\mu} \bar{\nabla}_{\mu} n=-\mathfrak{D}(\bar{\nabla} n)^{2} /\left(m n_{m}\right)$ where $(\bar{\nabla} n)^{2}=-\perp^{\mu \nu} \bar{\nabla}_{\mu} n \bar{\nabla}_{\nu} n$. So that eq. (3.58) is in fact a nonlinear diffusion equation.

Let us now turn to the identification of the imperfect part of the EMT, which can be done by comparing with the conventional gradient expansion performed in relativistic thermo/hydrodynamics. As we have already mentioned in our discussion of the EMT in section 3.4, it is necessary to solve the equation of motion for the scalar field in order to express the EMT purely through variables on the Cauchy hypersurface. In doing so, we rewrite the EMT in the effective form of a gradient expansion with $\kappa$ playing the role of the expansion parameter. Substituting the equation of motion (3.53) and the expression for the particle current in fluid variables (3.36) into the expression for the pressure (3.31) gives an EMT of the form

$$
T_{\mu \nu}=\mathcal{E} u_{\mu} u_{\nu}-(\widetilde{P}-\zeta \theta) \perp_{\mu \nu}+u_{\mu} q_{\nu}+u_{\nu} q_{\mu}+\mathcal{O}\left(\kappa^{2}\right),
$$

where we now have a pressure term corrected for the first-oder terms in this expansion,

$$
\widetilde{P} \equiv P+\frac{\kappa}{D} \mathcal{E}_{\phi}
$$

and there appears a bulk viscosity-like term with a would-be viscosity coefficient

$$
\zeta \equiv-D^{-1} \kappa P_{m}+\mathcal{O}\left(\kappa^{2}\right)=-\kappa\left(\frac{n}{n_{m}}\right)+\mathcal{O}\left(\kappa^{2}\right),
$$

and we have neglected the terms second-order in the diffusivity $\kappa$. We can compare this expression with the sound speed that we have obtained in [56, Eq. 3.20] for the cosmological background. ${ }^{14}$ Again dropping all terms of order $\kappa^{2}$, we can conclude that when the correction from kinetic braiding is small, the effective bulk viscosity,

$$
\zeta=-\kappa m c_{\mathrm{s}}^{2}+\mathcal{O}\left(\kappa^{2}\right) .
$$

However, it is important to bear in mind that this type of bulk viscosity differs from the usual notion defined in terms of the conventional gradient expansion. Indeed, in this expansion an important assumption is that the energy density does not receive any corrections

\footnotetext{
${ }^{14}$ As we show in our forthcoming work ref. [77], the sound speed on general backgrounds is more complicated but gives the same results at the level of precision required in this discussion.
} 
involving gradients of potentials or velocities. In our fluid, however, we do have a contribution to $\mathcal{E}$ proportional to $\theta$. Hence, the only way to appropriately compare to the language of the gradient expansion is to perform a redefinition of what is meant by the hydrodynamic potentials, order by order in $\kappa$. Thus, one has to introduce a new chemical potential

$$
\hat{m}=\hat{m}(m, \theta),
$$

in such a way as to keep the form that is the energy density unchanged between the zeroth and first orders in the expansion,

$$
\mathcal{E}_{0}(\hat{m}) \equiv \mathcal{E}(m, \theta)=\mathcal{E}_{0}(m)+\kappa m \theta,
$$

where $\mathcal{E}_{0}=m P_{m}-P$ is the k-essence energy density. All quantities are supposed to be expressed now in terms of $\hat{m}$. At leading order, we therefore have

$$
\hat{m} \approx m+\frac{\kappa m \theta}{\mathcal{E}_{0, m}}=m+\frac{\kappa \theta}{D} .
$$

the charge density $n$ becomes a function of $\hat{m}$ only, up to first order in $\kappa$

$$
n(m)=n_{0}(m)+\kappa \theta=n_{0}(\hat{m})+\mathcal{O}\left(\kappa^{2}\right) .
$$

This allows us to identify this modified chemical potential with the definition

$$
\hat{m}=\frac{\partial \mathcal{E}}{\partial n},
$$

which is valid to the same order up to same order without the need to specify that the derivative is taken at constant volume. The pressure using the new potential becomes

$$
\widetilde{P}(\hat{m})=\widetilde{P}(m)+\kappa m c_{\mathrm{s}}^{2} \theta .
$$

When combined with the bulk viscosity term we obtained in eq. (3.66), we can see that the contributions proportional to the expansion $\theta$ cancel out, and therefore the correct identification for the bulk viscosity of this fluid is

$$
\zeta_{\text {grad exp }}=0 .
$$

This is in tune with the fact that no actual dissipation takes place, as indeed it must not in the case of a theory arising from an action principle. ${ }^{15}$

Having said that, we must emphasise that the EMT does depend on the expansion $\theta$ : even the energy density does (in the original variables). This corresponds to a kind of "viscous" behaviour: a (perhaps new) type of nontrivial response to expansion that is not dissipative. Hence, it still seems appropriate to call this a viscid fluid, even if the conventionally defined linear bulk and shear viscosity coefficients vanish.

Taking as a measure of this type of viscosity the coefficient defined above in eq. (3.66), we can see that there is also a relation between viscosity and diffusion in this fluid:

$$
\zeta=\mathfrak{D} n m .
$$

\footnotetext{
${ }^{15}$ The same redefinitions of the chemical potential (3.67), particle number (3.70), energy density (3.68) and pressure (3.72) were recently rederived in the work [79], c.f. equations (A9)-(A12).
} 


\section{Equilibrium and vacuum configurations}

In this section, we will discuss the conditions on the fluid that are implied by the requirement that it is in equilibrium. As we will show here, the coupling of the braided fluid to gravity imposes an additional condition on the braided fluid compared to k-essence.

Let us assume a non-vanishing number of particles in the fluid, $n \neq 0$. In equilibrium in a stationary gravitational field with a timelike Killing vector $\xi_{\mu}$, the quantity which is constant throughout a system (in our case - on the spacelike hypersurfaces $\Sigma_{\phi}$ ) is not the locally measured chemical potential $m$ but

$$
m \sqrt{g_{\mu \nu} \xi^{\mu} \xi^{\nu}}=\text { const } .
$$

This is sometimes called Tolman-Klein condition, see ref. [80] and ref. [81, §27, p. 77].

In the discussion here we will concentrate on the shift-symmetric case. Firstly, since $\phi$ is an intrinsic clock and in equilibrium no physical observables should depend on this intrinsic clock explicitly. Secondly, comoving observables like $\mathcal{E}, m$, etc. should be independent of comoving time, e.g. $\dot{\mathcal{E}}=u^{\lambda} \nabla_{\lambda} \mathcal{E}=0$. Since we are building our description on the assumption that $m=\dot{\phi} \neq 0$, it better be the case that $\phi$ is not an observable, at least in equilibrium.

The requirement that observables be independent of comoving time implies that the Killing vector $\xi_{\mu} \propto u_{\mu}$. Since $u^{\mu}$ is hypersurface orthogonal by definition, this implies in fact that the equilibrium configuration is not just stationary, but also static. To satisfy eq. (4.1) in an arbitrary curved spacetime automatically — without new conditions - we have to assume that the coefficient of proportionality is $m^{-1}$ so that

$$
\text { in equilibrium } \quad \xi_{\mu}=\frac{u_{\mu}}{m} \text { is a Killing vector. }
$$

Therefore

$$
\nabla_{\nu} \xi_{\mu}+\nabla_{\mu} \xi_{\nu}=\frac{2 \mathcal{K}_{\mu \nu}}{m}-\frac{2 u_{\mu} u_{\nu}}{m^{2}} \dot{m}=0
$$

a projection of which gives

$$
\dot{m}=0 \text { and } \mathcal{K}_{\mu \nu}=0 .
$$

In particular, the expansion must vanish, $\theta=\mathcal{K}_{\mu}^{\mu}=0$. Thus we have shown that, in equilibrium, the motion of the fluid should be rigid in the Born sense: $\mathcal{K}_{\mu \nu}=0$. This is the standard requirement, see e.g. ref. [82].

We can also consider situations where more than one type of matter is present. Since $\xi^{\mu}$ is the only time-like Killing vector, the velocity of the external fluid must lie along $\xi^{\mu}$, and since the velocities are normalised $-u_{\text {ext }}^{\mu}=u^{\mu}$. The Tolman-Klein condition eq. (4.1) then implies chemical potentials of all the constituents must be equal, $m_{\text {ext }}=m$.

As we have already mentioned, the chemical potential $m$ for the system in an external field can be space dependent even in equilibrium. Thus the acceleration does not necessarily vanish. However, this is a result of the fact that a non-zero acceleration implies that $u^{\mu}$ is not tangent to a geodesic: the energy flux in equilibrium appears since the LRF is noninertial. One should stress, however, that the time dependence of the acceleration does 
vanish. The appropriate measure of the time dependence of a vector in the Lie derivative, and in this, case

$$
£_{\xi} a_{\mu}=a^{\lambda} \nabla_{\mu}\left(\frac{u^{\lambda}}{m}\right)+\frac{u^{\lambda}}{m} \nabla_{\lambda} a_{\mu}=0 .
$$

In general, to find the equilibrium configuration as a function on space, one would need to apply the equilibrium conditions eq. (4.4) to the Raychaudhuri equations and Einstein equations and solve this system. This is potentially a rich problem and lies outside of the scope of this work. Nonetheless, let us elucidate one significant difference between $\mathrm{k}$-essence and the braided fluid. Under the equilibrium conditions, the equation of motion for k-essence is trivial and does not supply any new information. However, in the case of the braided fluid, the equation of motion (3.46) reduces to a new constraint

$$
\kappa R_{\mu \nu} u^{\mu} u^{\nu}=-\kappa_{m} m a^{2}
$$

which, using the Einstein equations, becomes

$$
\mathcal{E}+3 P+\mathcal{E}_{\text {ext }}+3 P_{\text {ext }}=-2(\ln \kappa)^{\prime} a^{2},
$$

where the prime denotes differentiation with respect to $\ln m$ and the quantities with the subscript external are any potential contributions to the EMT coming from matter external to the braided scalar. Let us just say that this constraint imposes a condition on the equation of state of the braided scalar in equilibrium. In the extreme case of a constant diffusivity $\kappa$, this constraint implies that the equation of state is such that the scalar arranges itself in a configuration which screens the gravitational effect of the mass of the total EMT. In particular, when there is no external matter, the equation of state in equilibrium is $w=-1 / 3$. This is another facet of the monitoring behaviour present in the cosmological solutions which we discussed in detail in ref. [56].

Let us now briefly mention vacuum configurations where the charge density vanishes, $n=0$. In ref. [56] we showed that for the shift-symmetric theories with kinetic gravity braiding there exist such non-trivial vacuum solutions and that they are dynamical attractors in an expanding universe. As one can see from eq. (3.15), the vanishing of the shift-charge implies that the motion is restricted to obey

$$
\theta(m)=-\frac{P_{m}}{\kappa} .
$$

The structure of these configurations is potentially even richer than that of equilibrium configurations and this issue deserves a further detailed analysis going far beyond of the scope of this paper.

\section{How unnaturally complicated could it be?}

One really interesting example of how unexpectedly complicated and unnatural the function $K(X)$ can be is provided by the noninteracting relativistic degenerate fermions with mass 
$m_{\mathrm{e}}$ and chemical potential $m$, see e.g. [81, $\S 61$, p. 180]. In equilibrium, we have

$$
\begin{aligned}
n & =\frac{m_{\mathrm{e}}^{3}}{3 \pi^{2}} \sinh ^{3} \frac{\xi}{4}, \\
P & =\frac{m_{\mathrm{e}}^{4}}{32 \pi^{2}}\left(\frac{1}{3} \sinh \xi-\frac{8}{3} \sinh \frac{\xi}{2}+\xi\right), \\
\mathcal{E} & =\frac{m_{\mathrm{e}}^{4}}{32 \pi^{2}}(\sinh \xi-\xi), \\
m & =m_{\mathrm{e}} \cosh \frac{\xi}{4},
\end{aligned}
$$

where $\xi$ is related to Fermi momentum $p_{F}$ as

$$
\xi=4 \operatorname{arcsinh} \frac{p_{F}}{m_{\mathrm{e}}} .
$$

First of all, one can check that the Euler relation, eq. (3.37), indeed holds. Further, we can re-express the pressure through the chemical potential $m$ so that we obtain

$$
P(m)=\frac{m_{\mathrm{e}}^{4}}{12 \pi^{2}}\left[\frac{3}{2} \ln \left[\frac{m}{m_{\mathrm{e}}}+\sqrt{\left(\frac{m}{m_{\mathrm{e}}}\right)^{2}-1}\right]+\sqrt{\left(\frac{m}{m_{\mathrm{e}}}\right)^{4}-\left(\frac{m}{m_{\mathrm{e}}}\right)^{2}}\left[\left(\frac{m}{m_{\mathrm{e}}}\right)^{2}-\frac{5}{2}\right]\right] .
$$

If we restrict our attention to the simplest motion - vorticity-free - we have to follow Schutz [63] and the identification used in this work, eq. (3.22), and substitute $m=\sqrt{2 X}=$ $\sqrt{(\partial \phi)^{2}}$ into this formula for the pressure to obtain the Lagrangian. As the result of this procedure, we get a rather unusual scalar field theory of the k-essence type:

$$
P(\partial \phi)=\frac{m_{\mathrm{e}}^{4}}{12 \pi^{2}}\left[\frac{3}{2} \ln \left[\sqrt{\frac{(\partial \phi)^{2}}{m_{\mathrm{e}}^{2}}}+\sqrt{\frac{(\partial \phi)^{2}}{m_{\mathrm{e}}^{2}}-1}\right]+\sqrt{\frac{(\partial \phi)^{4}}{m_{\mathrm{e}}^{4}}-\frac{(\partial \phi)^{2}}{m_{\mathrm{e}}^{2}}}\left[\frac{(\partial \phi)^{2}}{m_{\mathrm{e}}^{2}}-\frac{5}{2}\right]\right] .
$$

Note that this Lagrangian describes a simple physical system and the incorporation of any interaction would only serve to make the structure more complicated. Moreover, this Lagrangian does not have a well defined vacuum limit at $(\partial \phi)^{2} \rightarrow 0$. This is the manifestation of the simple physical fact that the fermion chemical potential can never be smaller than the mass of the fermion $m_{\mathrm{e}}$. Even in the limit $(\partial \phi)^{2} \rightarrow m_{\mathrm{e}}^{2}$ the asymptotic is not analytic:

$$
P(\partial \phi) \simeq \frac{4 \sqrt{2}}{15 \pi^{2}} m_{\mathrm{e}}^{4}\left(\frac{\sqrt{(\partial \phi)^{2}}}{m_{\mathrm{e}}}-1\right)^{5 / 2} .
$$

Observe that the fundamental degrees of freedom in this system are fermions which have nothing to do with the effective bosonic field $\phi$. Moreover, the Lagrangian does not appear to be technically natural and the only free parameter $m_{\mathrm{e}}$ does not correspond to the actual strong-coupling scale. One could expect that the latter is governed by the scale of particle separation, $n^{1 / 3}$, which can be exponentially larger than $m_{\mathrm{e}}$.

It is important to stress here that the Lagrangian eq. (5.5) does not model all the degrees of freedom which exist in an ideal Fermi gas. In particular, it does not contain 
the zero sound, which is a non-equilibrium propagating mode. ${ }^{16}$ In ideal Fermi gases this mode dominates at low temperature, while the adiabatic hydrodynamic mode modeled by eq. (5.5) is actually suppressed. However, the addition of even a small attractive interaction allows the adiabatic mode to propagate [83, p. 163]. Adding such a small attractive interaction should have only a small effect on the equation of state of the fluid, since it only affects those modes lying close to the Fermi surface. On the other hand, we do not know whether there exist real examples of fluids where the hydrodynamic description we are using above would dominate over the non-equilibrium zero-sound modes. However, e.g. the modeling of ultra-high-density neutron stars is usually performed this hydrodynamic approximation.

Having seen the level of complexity allowed even for the simplest systems one can ask what are the natural structures of the function $G(X)$ ? The simplest option for the diffusivity $\kappa$ is just to be constant. This corresponds to

$$
G(X)=\frac{\kappa}{2} \ln X, \text { for } \kappa=\text { const } .
$$

Another option would be to declare the diffusion coefficient $\mathfrak{D}$ to be constant. This would correspond to $G$ 's and $K$ 's being connected as

$$
G(X)=-\mathfrak{D} \sqrt{2 X} K_{X}, \text { for } \mathfrak{D}=\text { const },
$$

where we have omitted a constant of integration. In this case, $G$ is just proportional to the charge density in equilibrium. However, as the simple physical example with fermions teaches us, Nature may not enjoy such structural simplicity at all.

\section{Discussion and future directions}

In this paper, we used a hydrodynamical language to describe the dynamics of theories with kinetic gravity braiding. This fluid picture turns out to be extremely useful: it provides an intuitively clear physical meaning to otherwise obscure combinations of derivatives of the field and the Lagrangian. This drastically simplifies notation allowing a much better understanding of the system. As is well known, finding the correct variables in many cases provides an easy path to the solution. Moreover, this notation clarifies the information encoded in the free functions $K(\phi, X)$ and $G(\phi, X)$, making it possible to construct these theories based on physical principles and not solely from a naive naturalness.

The key feature of our fluid, is the dependence of the charge density and, correspondingly, the energy density on the expansion $\theta$. This allows for the presence of bulk viscositylike effects and diffusion without any dissipation. This novel property, to our best knowledge, had not been considered in the literature of hydrodynamics hitherto.

We would like to mention that our identification of shift charges with particles and, therefore, of the proper-time gradient of the scalar field with the chemical potential is not actually unique. In fact, we could also have assumed that these charges correspond to entropy. From the first law of thermodynamics, it follows that this alternative identification could be obtained by the exchange $n \rightarrow s$ and $m \rightarrow T$, where $T$ is the temperature. We

\footnotetext{
${ }^{16}$ We thank Gregory Gabadadze, Massimo Porrati and Alberto Nicolis for discussing this with us.
} 
find that the identification presented in the paper is more satisfactory. In the case with entropy, even for a perfect fluid EMT, a non-shift symmetric Lagrangian would imply that entropy is not conserved. Moreover, even in the shift-symmetric case, our diffusion flux would correspond to a heat flux, but one which would nonetheless conserve entropy.

The equation of motion for the scalar is a highly nonlinear partial differential equation of the Ampère-Monge type. This class of equations belongs to the frontiers of current research in mathematics. In fact, contrary to the quasilinear case, it is not even clear under which conditions the Cauchy problem is well posed. It may happen that this fluid picture could aid in developing the understanding of this problem. In the forthcoming paper, ref. [77] we make a first step in this direction by discussing the high-frequency stability and acoustic geometry in these theories.

As we have alluded to already, our hydrodynamical picture owes a lot to ref. [63], where perfect fluids with vorticity and thermal effects are described through a Lagrangian. It would be interesting to investigate whether a non-pathological and meaningful generalisation of perfect fluids with vorticity and thermal properties is possible along the lines discussed in our paper. We believe that for this purpose the Lagrangian $\tilde{\mathcal{P}}(\phi, m, \theta)$ from eq. (B.10) could be rather suitable. In ref. [63] the author had already conjectured: "It may also be possible to extend this work to viscous fluids...". In fact, the current paper can be considered as a first step in this direction. Moreover, one could consider similar generalisations in the framework of ref. [84]; for a recent development in this direction see e.g. refs. $[85,86]$.

In a rather natural extension, one could employ the formalism developed in this paper to consider the hydrodynamical properties of the more general galileons and theories such as those of ref. [53]. There is, however, a potential difficulty in interpretation which is related to a necessary appearance of curvature terms in the action, see ref. [26, 27].

Further, one should not forget that boundary terms are in fact unavoidable for the proper formulation of the action principle in theories with higher derivatives, see e.g. [87, 88]. It would be very interesting and important to find these terms and their possible fluid interpretation in theories with kinetic gravity braiding.

Having in mind the higher-derivative structure of the theory, it is interesting to speculate how one could change the fluid picture using constraints as in ref. [89]. It seems that, in particular, one could obtain a kinetically braided dust - a braided fluid moving along geodesics. Another option would be to constrain the expansion in the action and obtain in that way a braided incompressible fluid with diffusion.

Finally, the fluid presented here appears to naturally act so as to screen the gravitational effects of matter when it is in an equilibrium configuration. This is another manifestation of the monitoring effect that we discussed for the cosmological solutions of these models in ref. [56]. Such phenomenology would clearly be important in cosmology during non-linear structure formation. Could the equilibrium configurations which we have described here provide a model for dark-matter haloes which significantly deviates from the standard CDM paradigm?

To conclude, we are in these days witnessing a revival of interest and a rather exciting series of developments in our understanding of non-canonical field theories and hydrody- 
namics. The bestiary of models used in cosmological models has grown and we are in the process of trying to understand the implications. Eventually, these models will need to stand up to observational tests, whether in cosmology or possibly even in some condensedmatter systems, and we are in the process of trying to understand whether they could have any completely new signatures for which to hunt.

\section{Acknowledgments}

It is a pleasure to thank Luca Amendola, Eugeny Babichev, Andrei Barvinski, Ram Brustein, Gerhard Buchalla, Jorge Casalderrey-Solana, Alexander Dolgov, Sergei Dubovsky, Gia Dvali, Andrei Frolov, Gregory Gabadadze, Jaume Garriga, Cristiano Germani, Dmitry Gorbunov, Andrei Gruzinov, Bhilahari Jeevanesan, Andrei Khmelnitsky, Maxim Libanov, Viatcheslav Mukhanov, Shinji Mukohyama, Alberto Nicolis, Massimo Porrati, Valery Rubakov, Mikhail Shaposhnikov, Sergey Sibiryakov, Christof Wetterich for very useful discussions and criticisms. We extend special gratitude to Cédric Deffayet for his comments throughout the preparation of this work and for the collaboration on initial stages of this project. A significant part of this work was completed at the time when I. S. and A. V. were enjoying the support of the James Arthur Fellowship at the Center for Cosmology and Particle Physics, New York University. I. S. and A. V. would like to thank for this support and for the lively atmosphere at the Center. In the final stages of this project, I. S. was supported by the DFG through TRR33 "The Dark Universe". I. S. and A. V. would also like to thank the CERN Theory Division for their hospitality during the preparation of this manuscript. This visit of A. V. to CERN was supported through a grant of the David and Lucile Packard Foundation. A. V. is also thankful to the organizers of the workshop Gravity and Cosmology 2010 at the Yukawa Institute for Theoretical Physics, Kyoto University for their hospitality and financial support during the intermediate stages of this project, many results of which were presented and discussed during the workshop.

\section{A Choice of frames}

The choice of $u^{\mu}$ defined in eq. (3.1) as a velocity for the frame is special in that it is explicitly vorticity free as a result of this velocity's being parallel to the gradient of $\phi$. This means that we are permitted to perform a foliation of the space-time using $\phi$ as an internal clock and the surfaces $\Sigma_{\phi}: \phi=$ const as our spatial hypersurfaces. This particular foliation is then a natural candidate for being a Cauchy surface. In the follow-up work ref. [77], we discuss the requirements on the possible general fluid configurations which ensure that this particular choice of spatial hypersurfaces indeed provides a surface on which initial values can be supplied in the usual unconstrained fashion and one can honestly describe the scalar field as a fluid.

Using a general frame moving with some other velocity $U^{\mu}$ we could have defined the pressure, energy density and energy flow using equations (3.30), (3.32) and (3.33) with the substitution $u^{\mu} \rightarrow U^{\mu}$. In these variables the EMT would be

$$
T_{\mu \nu}=\epsilon_{U} U_{\mu} U_{\nu}-\perp_{\mu \nu}^{U} \mathcal{P}_{U}+q_{\mu}^{U} U_{\nu}+q_{\nu}^{U} U_{\mu}+\pi_{\mu \nu}
$$


where

$$
\pi_{\mu \nu}=\left(\perp_{\mu \alpha}^{U} \perp_{\nu \beta}^{U}-\frac{1}{3} \perp_{\mu \nu}^{U} \perp_{\alpha \beta}^{U}\right) T^{\alpha \beta},
$$

is the shear-stress tensor. Note that in principle we could perform this decomposition for an arbitrary EMT. Generically, none of the shear, rotation or vorticity will vanish in such a frame and the shear-stress tensor will contain terms with $\mathcal{P}$ and, correspondingly, $\dot{m}$. Hence, for general initial data, $\pi_{\mu \nu}$ will not vanish. In particular, this is the case for the two most popular frames used in the analysis of standard theories of imperfect fluids: the Eckart frame, where the frame moves with the particle flow (so that $U_{\mathrm{E}}^{\mu}=J^{\mu} / \sqrt{J^{\lambda} J_{\lambda}}$ ), and the Landau-Lifshitz frame, which moves together with the energy so that $T_{\nu}^{\mu} U_{\mathrm{LL}}^{\nu}=$ $\epsilon_{\mathrm{LL}} U_{\mathrm{LL}}^{\mu}$. It could well be that these frames may not even exist for some otherwise reasonable initial data.

One should note, however, that up to (and including) the first order in $\kappa$, these two frames coincide, since the actual heat flux is absent, see [82, Eq. (2), p. 312]. Moreover, up to this order, the energy-momentum tensor in this Landau-Lifshitz frame takes the perfect-fluid form. And indeed, up to and including the first order in $\kappa$ we are then dealing with a perfect fluid. However, once higher-order corrections are included, we see that the price paid for this frame change is that the fluid flow is now no longer vorticity free, with corrections also coming at second order in $\kappa$. We show this explicitly below.

Let us for simplicity concentrate on the shift-symmetric case. In the Eckart frame (suppose it exists so that (3.19) holds), the shift-charge density is

$$
n_{\mathrm{E}}=\sqrt{J_{\mu} J^{\mu}}=n+\mathcal{O}\left(\kappa^{2}\right)=P_{m}+\kappa \theta+\mathcal{O}\left(\kappa^{2}\right) .
$$

The 4-velocity of this frame is

$$
U_{\mathrm{E}}^{\mu}=J^{\mu} / n_{\mathrm{E}}=u^{\mu}+\mathcal{O}(\kappa) .
$$

Now let us calculate the kinematical rotation vector for the Eckart frame, see e.g. [90]:

$$
\omega^{\mu}\left(U_{\mathrm{E}}\right) \equiv \varepsilon^{\alpha \beta \gamma \mu}\left(\nabla_{\alpha} U_{\mathrm{E} \beta}\right) U_{\mathrm{E} \gamma}=\omega^{\mu}(J) / n_{\mathrm{E}}^{2} .
$$

This kinematical rotation vector is related to the previously mentioned rotation tensor or twist in this frame $w_{\mu \nu}^{\mathrm{E}}=1 / 2\left(\perp_{\mu \alpha}^{U} \nabla^{\alpha} U_{\mathrm{E} \nu}-\perp_{\nu \alpha}^{U} \nabla^{\alpha} U_{\mathrm{E} \mu}\right)$ in the following way:

$$
\omega^{\mu}\left(U_{\mathrm{E}}\right)=\varepsilon^{\alpha \beta \gamma \mu}\left(\perp_{\alpha \lambda}^{U} \nabla^{\lambda} U_{\mathrm{E} \beta}\right) U_{\mathrm{E} \gamma}=\varepsilon^{\alpha \beta \gamma \mu} w_{\alpha \beta}^{\mathrm{E}} U_{\mathrm{E} \gamma} .
$$

Further for $\omega^{\mu}(J)$ we have

$$
\omega^{\mu}(J)=\varepsilon^{\alpha \beta \gamma \mu} \nabla_{\alpha} J_{\beta} J_{\gamma} .
$$

Some tedious but straightforward algebra yields the result that the vorticity in the Eckhart frame, in terms of the variables in our chosen local rest frame defined by the gradient of $\phi$,

$$
\omega^{\mu}\left(U_{\mathrm{E}}\right)=\frac{\kappa^{2}}{n_{\mathrm{E}}^{2}} \epsilon^{\alpha \beta \gamma \mu} S_{\alpha} u_{\beta} a_{\gamma},
$$


with

$$
S_{\alpha} \equiv \frac{\bar{\nabla}_{\alpha} \dot{m}}{m}-\bar{\nabla}_{\alpha} \theta
$$

Thus the kinematical rotation vector $\omega^{\mu}\left(U_{\mathrm{E}}\right)$ is $\mathcal{O}\left(\kappa^{2}\right)$ and vanishes on equilibrium configurations. In order to calculate it, one must solve the equation of motion (3.53). Nonetheless, for general initial data, neither $\bar{\nabla}_{\alpha} \dot{m}$ nor $\bar{\nabla}_{\alpha} \theta$ are parallel to $a_{\mu}$, therefore $\omega^{\mu}\left(U_{\mathrm{E}}\right)$ does not vanish. Nonvanishing vorticity implies (see Frobenius theorem) that the Eckart frame is not suitable for the formulation of the Cauchy problem, because $U_{\mathrm{E}}^{\mu}$ is not hypersurface-orthogonal.

Using the variables we defined in section 3.7, we can also rewrite the full EMT

$$
T_{\mu \nu}=\left(\mathcal{E}_{0}+\widetilde{P}\right) u_{\mu} u_{\nu}-g_{\mu \nu} \widetilde{P}-\kappa \hat{m}\left(u_{\mu} a_{\nu}+u_{\nu} a_{\mu}\right)+\mathcal{O}\left(\kappa^{2}\right) .
$$

We can now perform a diagonalization into the Landau-Lifshitz frame by boosting the velocity $u_{\mu}$ along $a_{\mu}$,

$$
\begin{aligned}
U_{\mathrm{LL} \mu} & =u_{\mu}-\alpha a_{\mu}+\mathcal{O}\left(\kappa^{2}\right) \\
\widetilde{a}_{\mu} & =-\alpha u_{\mu}+a_{\mu}+\mathcal{O}\left(\kappa^{2}\right),
\end{aligned}
$$

with $\alpha \equiv \kappa \hat{m} /\left(\mathcal{E}_{0}+\widetilde{P}\right)$. Rewriting eq. (A.10) using the new velocity, we obtain an EMT of perfect-fluid form,

$$
T_{\mu \nu}=\left(\mathcal{E}_{0}+\widetilde{P}\right) U_{\mathrm{LL} \mu} U_{\mathrm{LL} \nu}-g_{\mu \nu} \widetilde{P}+\mathcal{O}\left(\kappa^{2}\right) .
$$

However, at second order in $\kappa$, deviations away from a perfect fluid reappear in this frame in the form of a non-vanishing anisotropic stress proportional to $\widetilde{a}_{\mu} \widetilde{a}_{\nu}$.

The formulation of the theory in terms of the field $\phi$ provides a natural reference frame which is neither the Landau-Lifshitz frame nor the Eckart frame: the bulk velocity $u^{\mu}$ is neither the velocity of energy nor the velocity of particles. Contrary to the usual imperfect fluids, in this frame, the energy density and particle density contain first derivatives of this four-velocity. Moreover, the pressure contains the second time derivative of the scalar field. It is also in this reference frame where the theory has a cosmological solution and reduces to perfect-fluid configurations. Finally, the shift-charge/particle-number current depends on initial data only and it seems that there are no general restrictions on the functions $G(\phi, X)$ and $K(\phi, X)$ such that the current $J^{\mu}$ is timelike for all admissible initial data.

\section{B Action without second time derivatives}

Let us start from classical mechanics and consider the following one-dimensional version of the system represented by our action (2.1),

$$
S=\int \mathrm{d} t(K(q, \dot{q})+G(q, \dot{q}) \ddot{q}) .
$$

Now we can add a total derivative to this action without changing the equation of motion so that:

$$
\ddot{q} G(q, \dot{q})+\frac{d}{d t} f(q, \dot{q})=\ddot{q}\left(G+f_{, \dot{q}}\right)+f_{, q} \dot{q} .
$$


We can eliminate the second derivative $\ddot{q}$ if we take

$$
f=-\int^{\dot{q}} d v G(q, v)
$$

in which case the new, equivalent, Lagrange function without higher derivatives is

$$
L_{1}(q, \dot{q})=K(q, \dot{q})-\dot{q} \int^{\dot{q}} d v G_{, q}(q, v) .
$$

Now let us see how one can eliminate the second time derivative for our kinetically braided scalar field $\phi$. Let us use the Lagrangian (2.3) and write the action in the comoving frame in the form

$$
S_{\phi}=\int \mathrm{d}^{3} x \int \mathrm{d} \tau \sqrt{-g}(P-\kappa \dot{m})
$$

where now $g=g^{\tau \tau} \perp$ with $\perp \equiv \operatorname{det} \perp_{\mu \nu}$ and

$$
g^{\tau \tau}=g^{\mu \nu} \partial_{\mu} \tau \partial_{\nu} \tau
$$

Recalling the definition of the effective mass $m$ eq. (3.22) we have

$$
\mathrm{d} \tau=\frac{\mathrm{d} \phi}{m}, \text { so that } g^{\tau \tau}=\frac{g^{\mu \nu} \partial_{\mu} \phi \partial_{\nu} \phi}{m^{2}}=1 .
$$

Now we can add an arbitrary total derivative $\mathrm{d}(f \sqrt{-\perp}) / \mathrm{d} \tau$ without changing physics so that

$$
S_{\phi 1}=\int \mathrm{d}^{3} x \int \mathrm{d} \tau \sqrt{-\perp}\left(P-\kappa \dot{m}+f_{\phi} m+f \frac{\mathrm{d}}{\mathrm{d} \tau} \ln \sqrt{-\perp}+f_{m} \dot{m}\right) .
$$

Therefore for

$$
f(\phi, m)=\int^{m} d m^{\prime} \kappa\left(m^{\prime}, \phi\right)
$$

we eliminate $\dot{m}=\ddot{\phi}$ from the action. Further, we notice that the expansion can be expressed as

$$
\theta=\frac{d}{d \tau} \ln \sqrt{-\perp}
$$

Thus we obtain an equivalent new Lagrangian $\tilde{\mathcal{P}}$ without second time derivatives in comoving coordinates

$$
S_{\phi 1}=\int \mathrm{d}^{4} x \sqrt{-g}\left(P+m f_{\phi}+f \theta\right)=\int \mathrm{d}^{4} x \sqrt{-g} \tilde{\mathcal{P}}(\phi, m, \theta) .
$$

Finally one can check that the difference between Lagrangians is indeed a total derivative:

$$
\tilde{\mathcal{P}}-\mathcal{P}=\kappa \dot{m}+m \int^{m} d m^{\prime} \kappa_{\phi}+\theta \int^{m} d m^{\prime} \kappa=\nabla_{\mu}\left(\int^{m} d m^{\prime} \kappa\left(m^{\prime}, \phi\right) u^{\mu}\right) .
$$

Of course if we were to change the coordinates away from this natural LRF frame, $\tilde{\mathcal{P}}$ would again contain second time derivatives. 
Open Access. This article is distributed under the terms of the Creative Commons Attribution Noncommercial License which permits any noncommercial use, distribution, and reproduction in any medium, provided the original author(s) and source are credited.

\section{References}

[1] C. Armendariz-Picon, V.F. Mukhanov and P.J. Steinhardt, Essentials of $k$ essence, Phys. Rev. D 63 (2001) 103510 [astro-ph/0006373] [INSPIRE].

[2] C. Armendariz-Picon, V.F. Mukhanov and P.J. Steinhardt, A dynamical solution to the problem of a small cosmological constant and late time cosmic acceleration, Phys. Rev. Lett. 85 (2000) 4438 [astro-ph/0004134] [INSPIRE].

[3] C. Armendariz-Picon, T. Damour and V.F. Mukhanov, $k$-inflation, Phys. Lett. B 458 (1999) 209 [hep-th/9904075] [INSPIRE].

[4] J. Garriga and V.F. Mukhanov, Perturbations in k-inflation, Phys. Lett. B 458 (1999) 219 [hep-th/9904176] [INSPIRE].

[5] N. Arkani-Hamed, H.-C. Cheng, M.A. Luty and S. Mukohyama, Ghost condensation and a consistent infrared modification of gravity, JHEP 05 (2004) 074 [hep-th/0312099] [INSPIRE].

[6] R.J. Scherrer, Purely kinetic $k$-essence as unified dark matter, Phys. Rev. Lett. 93 (2004) 011301 [astro-ph/0402316] [inSPIRE].

[7] D. Giannakis and W. Hu, Kinetic unified dark matter, Phys. Rev. D 72 (2005) 063502 [astro-ph/0501423] [INSPIRE].

[8] C. Armendariz-Picon and E.A. Lim, Haloes of $k$-essence, JCAPA,0508,007. 20050508 (2005) 007 [astro-ph/0505207] [INSPIRE].

[9] D. Bertacca, S. Matarrese and M. Pietroni, Unified dark matter in scalar field cosmologies, Mod. Phys. Lett. A 22 (2007) 2893 [astro-ph/0703259] [INSPIRE].

[10] P. Creminelli, G. D'Amico, J. Norena and F. Vernizzi, The effective theory of quintessence: the $w<-1$ side unveiled, JCAP 02 (2009) 018 [arXiv:0811.0827] [INSPIRE].

[11] P. Creminelli, G. D'Amico, J. Norena, L. Senatore and F. Vernizzi, Spherical collapse in quintessence models with zero speed of sound, JCAP 03 (2010) 027 [arXiv:0911.2701] [INSPIRE].

[12] D. Bertacca, N. Bartolo and S. Matarrese, Unified dark matter scalar field models, Adv. Astron. 2010 (2010) 904379 [arXiv: 1008.0614] [INSPIRE].

[13] T. Furukawa, S. Yokoyama, K. Ichiki, N. Sugiyama and S. Mukohyama, Ghost dark matter, JCAP 05 (2010) 007 [arXiv: 1001.4634] [INSPIRE].

[14] G. Dvali, G. Gabadadze and M. Porrati, 4D gravity on a brane in $5 D$ Minkowski space, Phys. Lett. B 485 (2000) 208 [hep-th/0005016] [InSPIRE].

[15] G. Gabadadze, General relativity with an auxiliary dimension, Phys. Lett. B 681 (2009) 89 [arXiv:0908.1112] [INSPIRE].

[16] M.A. Luty, M. Porrati and R. Rattazzi, Strong interactions and stability in the DGP model, JHEP 09 (2003) 029 [hep-th/0303116] [INSPIRE].

[17] A. Nicolis and R. Rattazzi, Classical and quantum consistency of the DGP model, JHEP 06 (2004) 059 [hep-th/0404159] [INSPIRE]. 
[18] G. Gabadadze and A. Iglesias, (De)coupling limit of DGP, Phys. Lett. B 639 (2006) 88 [hep-th/0603199] [INSPIRE].

[19] E. Babichev, C. Deffayet and R. Ziour, k-mouflage gravity, Int. J. Mod. Phys. D 18 (2009) 2147 [arXiv:0905.2943] [InSPIRE].

[20] E. Babichev, C. Deffayet and R. Ziour, Recovering general relativity from massive gravity, Phys. Rev. Lett. 103 (2009) 201102 [arXiv:0907.4103] [INSPIRE].

[21] A. Nicolis, R. Rattazzi and E. Trincherini, The galileon as a local modification of gravity, Phys. Rev. D 79 (2009) 064036 [arXiv:0811.2197] [INSPIRE].

[22] A. Nicolis, R. Rattazzi and E. Trincherini, Energy's and amplitudes' positivity, JHEP 05 (2010) 095 [arXiv: 0912.4258] [INSPIRE].

[23] D. Fairlie, J. Govaerts and A. Morozov, Universal field equations with covariant solutions, Nucl. Phys. B 373 (1992) 214 [hep-th/9110022] [INSPIRE].

[24] D. Fairlie and J. Govaerts, Euler hierarchies and universal equations, J. Math. Phys. 33 (1992) 3543 [hep-th/9204074] [inSPIRE].

[25] D. Fairlie, Comments on Galileons, J. Phys. A 44 (2011) 305201 [arXiv:1102.1594] [INSPIRE].

[26] C. Deffayet, G. Esposito-Farese and A. Vikman, Covariant Galileon, Phys. Rev. D 79 (2009) 084003 [arXiv:0901.1314] [INSPIRE].

[27] C. Deffayet, S. Deser and G. Esposito-Farese, Generalized Galileons: all scalar models whose curved background extensions maintain second-order field equations and stress-tensors, Phys. Rev. D 80 (2009) 064015 [arXiv:0906.1967] [INSPIRE].

[28] N. Chow and J. Khoury, Galileon cosmology, Phys. Rev. D 80 (2009) 024037 [arXiv:0905.1325] [INSPIRE].

[29] F.P. Silva and K. Koyama, Self-accelerating universe in Galileon cosmology, Phys. Rev. D 80 (2009) 121301 [arXiv:0909.4538] [InSPIRE].

[30] T. Kobayashi, H. Tashiro and D. Suzuki, Evolution of linear cosmological perturbations and its observational implications in Galileon-type modified gravity, Phys. Rev. D 81 (2010) 063513 [arXiv:0912.4641] [INSPIRE].

[31] A. De Felice and S. Tsujikawa, Generalized Brans-Dicke theories, JCAP 07 (2010) 024 [arXiv: 1005.0868] [INSPIRE].

[32] A. De Felice, S. Mukohyama and S. Tsujikawa, Density perturbations in general modified gravitational theories, Phys. Rev. D 82 (2010) 023524 [arXiv: 1006.0281] [INSPIRE].

[33] P. Creminelli, A. Nicolis and E. Trincherini, Galilean genesis: an alternative to inflation, JCAP 11 (2010) 021 [arXiv: 1007.0027] [INSPIRE].

[34] A. De Felice and S. Tsujikawa, Cosmology of a covariant Galileon field, Phys. Rev. Lett. 105 (2010) 111301 [arXiv:1007.2700] [INSPIRE].

[35] R. Gannouji and M. Sami, Galileon gravity and its relevance to late time cosmic acceleration, Phys. Rev. D 82 (2010) 024011 [arXiv: 1004.2808] [INSPIRE].

[36] T. Kobayashi, Cosmic expansion and growth histories in Galileon scalar-tensor models of dark energy, Phys. Rev. D 81 (2010) 103533 [arXiv: 1003.3281] [INSPIRE].

[37] A. De Felice and S. Tsujikawa, Generalized Galileon cosmology, arXiv:1008.4236 [INSPIRE]. 
[38] S. Mizuno and K. Koyama, Primordial non-gaussianity from the DBI Galileons, Phys. Rev. D 82 (2010) 103518 [arXiv: 1009.0677] [InSPIRE].

[39] C. Burrage, C. de Rham, D. Seery and A.J. Tolley, Galileon inflation, JCAP 01 (2011) 014 [arXiv: 1009.2497] [INSPIRE].

[40] D.F. Mota, M. Sandstad and T. Zlosnik, Cosmology of the selfaccelerating third order Galileon, JHEP 12 (2010) 051 [arXiv:1009.6151] [INSPIRE].

[41] S. Nesseris, A. De Felice and S. Tsujikawa, Observational constraints on Galileon cosmology, Phys. Rev. D 82 (2010) 124054 [arXiv:1010.0407] [InSPIRE].

[42] P. Creminelli, G. D’Amico, M. Musso, J. Norena and E. Trincherini, Galilean symmetry in the effective theory of inflation: new shapes of non-gaussianity, JCAP 02 (2011) 006 [arXiv: 1011.3004] [INSPIRE].

[43] A. De Felice and S. Tsujikawa, Primordial non-gaussianities in general modified gravitational models of inflation, JCAP 04 (2011) 029 [arXiv:1103.1172] [INSPIRE].

[44] A. De Felice, R. Kase and S. Tsujikawa, Matter perturbations in Galileon cosmology, Phys. Rev. D 83 (2011) 043515 [arXiv:1011.6132] [InSPIRE].

[45] M. Wyman, Galilean-invariant scalar fields can strengthen gravitational lensing, Phys. Rev. Lett. 106 (2011) 201102 [arXiv:1101.1295] [INSPIRE].

[46] K. Hinterbichler, M. Trodden and D. Wesley, Multi-field Galileons and higher co-dimension branes, Phys. Rev. D 82 (2010) 124018 [arXiv: 1008.1305] [INSPIRE].

[47] A. Padilla, P.M. Saffin and S.-Y. Zhou, Bi-Galileon theory I: motivation and formulation, JHEP 12 (2010) 031 [arXiv:1007.5424] [INSPIRE].

[48] A. Padilla, P.M. Saffin and S.-Y. Zhou, Multi-Galileons, solitons and Derrick's theorem, Phys. Rev. D 83 (2011) 045009 [arXiv: 1008.0745] [INSPIRE].

[49] A. Padilla, P.M. Saffin and S.-Y. Zhou, Bi-Galileon theory II: phenomenology, JHEP 01 (2011) 099 [arXiv: 1008.3312] [INSPIRE].

[50] E. Babichev, Galileon accretion, Phys. Rev. D 83 (2011) 024008 [arXiv:1009.2921] [INSPIRE].

[51] K. Van Acoleyen and J. Van Doorsselaere, Galileons from Lovelock actions, Phys. Rev. D 83 (2011) 084025 [arXiv: 1102.0487] [InSPIRE].

[52] C. de Rham and A.J. Tolley, DBI and the Galileon reunited, JCAP 05 (2010) 015 [arXiv: 1003.5917] [INSPIRE].

[53] C. Deffayet, X. Gao, D. Steer and G. Zahariade, From k-essence to generalised Galileons, Phys. Rev. D 84 (2011) 064039 [arXiv:1103.3260] [inSPIRE].

[54] M. Andrews, K. Hinterbichler, J. Khoury and M. Trodden, Instabilities of spherical solutions with multiple Galileons and $\mathrm{SO}(N)$ symmetry, Phys. Rev. D 83 (2011) 044042 [arXiv: 1008.4128] [INSPIRE].

[55] G.L. Goon, K. Hinterbichler and M. Trodden, Stability and superluminality of spherical DBI Galileon solutions, Phys. Rev. D 83 (2011) 085015 [arXiv: 1008.4580] [INSPIRE].

[56] C. Deffayet, O. Pujolàs, I. Sawicki and A. Vikman, Imperfect dark energy from kinetic gravity braiding, JCAP 10 (2010) 026 [arXiv: 1008.0048] [INSPIRE]. 
[57] T. Kobayashi, M. Yamaguchi and J. Yokoyama, G-inflation: inflation driven by the Galileon field, Phys. Rev. Lett. 105 (2010) 231302 [arXiv: 1008.0603] [INSPIRE].

[58] R. Kimura and K. Yamamoto, Large scale structures in kinetic gravity braiding model that can be unbraided, JCAP 04 (2011) 025 [arXiv: 1011.2006] [INSPIRE].

[59] K. Kamada, T. Kobayashi, M. Yamaguchi and J. Yokoyama, Higgs G-inflation, Phys. Rev. D 83 (2011) 083515 [arXiv:1012.4238] [INSPIRE].

[60] J.-O. Gong and T. Tanaka, A covariant approach to general field space metric in multi-field inflation, JCAP 03 (2011) 015 [arXiv:1101.4809] [INSPIRE].

[61] T. Kobayashi, M. Yamaguchi and J. Yokoyama, Primordial non-gaussianity from G-inflation, Phys. Rev. D 83 (2011) 103524 [arXiv:1103.1740] [INSPIRE].

[62] A. Naruko and M. Sasaki, Conservation of the nonlinear curvature perturbation in generic single-field inflation, Class. Quant. Grav. 28 (2011) 072001 [arXiv:1101.3180] [INSPIRE].

[63] B.F. Schutz, Perfect fluids in general relativity: velocity potentials and a variational principle, Phys. Rev. D 2 (1970) 2762 [INSPIRE].

[64] S. Unnikrishnan and L. Sriramkumar, A note on perfect scalar fields, Phys. Rev. D 81 (2010) 103511 [arXiv:1002.0820] [inSPIRE].

[65] F. Arroja and M. Sasaki, A note on the equivalence of a barotropic perfect fluid with a k-essence scalar field, Phys. Rev. D 81 (2010) 107301 [arXiv: 1002.1376] [INSPIRE].

[66] L. D. Landau and E. M. Lifshitz, Course of theoretical physics. Volume 6: fluid mechanics, $2^{\text {nd }}$ edition, Pergamon Press, U.K. (1987).

[67] V. Moncrief, Stability of stationary, spherical accretion onto a Schwarzschild black hole, Astrophys. J. 235 (1980) 1038.

[68] A. Diez-Tejedor and A. FEinstein, Relativistic hydrodynamics with sources for cosmological k-fluids, Int. J. Mod. Phys. D 14 (2005) 1561 [gr-qc/0501101] [InSPIRE].

[69] N. Bilic, Thermodynamics of k-essence, Phys. Rev. D 78 (2008) 105012 [arXiv:0806.0642] [INSPIRE].

[70] N. Andersson and G. Comer, Relativistic fluid dynamics: physics for many different scales, Living Rev. Rel. 10 (2005) 1 [gr-qc/0605010] [INSPIRE].

[71] C. Misner, K. Thorne and J. Wheeler, Gravitation, W.H. Freeman \& Co., U.S.A. (1973).

[72] R. Akhoury, C.S. Gauthier and A. Vikman, Stationary configurations imply shift symmetry: no Bondi accretion for quintessence/k-essence, JHEP 03 (2009) 082 [arXiv:0811.1620] [INSPIRE].

[73] S. Nojiri and S.D. Odintsov, Inhomogeneous equation of state of the universe: phantom era, future singularity and crossing the phantom barrier, Phys. Rev. D 72 (2005) 023003 [hep-th/0505215] [INSPIRE].

[74] S. Capozziello, V. Cardone, E. Elizalde, S. Nojiri and S. Odintsov, Observational constraints on dark energy with generalized equations of state, Phys. Rev. D 73 (2006) 043512 [astro-ph/0508350] [INSPIRE].

[75] S. Nojiri and S.D. Odintsov, Introduction to modified gravity and gravitational alternative for dark energy, eConf C 0602061 (2006) 06 [hep-th/0601213] [INSPIRE]. 
[76] S.R. de Groot, W.A. van Leeuwen and C.G. van Weert, Relativistic kinetic theory: principles and applications, North-Holland Publishing Company, The Netherlands (1980).

[77] O. Pujolas, I. Sawicki and A. Vikman, Stability and acoustic metric in fluids with kinetic gravity braiding, in preparation.

[78] A. Vikman, Can dark energy evolve to the phantom?, Phys. Rev. D 71 (2005) 023515 [astro-ph/0407107] [INSPIRE].

[79] S. Dubovsky, L. Hui, A. Nicolis and D.T. Son, Effective field theory for hydrodynamics: thermodynamics and the derivative expansion, arXiv:1107.0731 [INSPIRE].

[80] O. Klein, On the thermodynamical equilibrium of fluids in gravitational fields, Rev. Mod. Phys. 21 (1949) 531.

[81] L.D. Landau and E.M. Lifshitz, Course of theoretical physics. Volume 5: statistical physics, part 1, Pergamon Press, U.K. (1980).

[82] W. Israel, Nonstationary irreversible thermodynamics: a causal relativistic theory, Annals Phys. 100 (1976) 310 [INSPIRE].

[83] L.D. Landau and E.M. Lifshitz, Course of theoretical physics. Volume 9: statistical physics, part 2, Pergamon Press, U.K. (1980).

[84] S. Dubovsky, T. Gregoire, A. Nicolis and R. Rattazzi, Null energy condition and superluminal propagation, JHEP 03 (2006) 025 [hep-th/0512260] [INSPIRE].

[85] A. Nicolis and D.T. Son, Hall viscosity from effective field theory, arXiv:1103.2137 [INSPIRE].

[86] S. Endlich, A. Nicolis, R. Rattazzi and J. Wang, The quantum mechanics of perfect fluids, JHEP 04 (2011) 102 [arXiv: 1011.6396] [INSPIRE].

[87] E. Dyer and K. Hinterbichler, Boundary terms and junction conditions for the DGP $\pi$-Lagrangian, JHEP 11 (2009) 059 [arXiv:0907.1691] [INSPIRE].

[88] E. Dyer and K. Hinterbichler, Boundary terms, variational principles and higher derivative modified gravity, Phys. Rev. D 79 (2009) 024028 [arXiv:0809.4033] [INSPIRE].

[89] E.A. Lim, I. Sawicki and A. Vikman, Dust of dark energy, JCAP 05 (2010) 012 [arXiv:1003.5751] [INSPIRE].

[90] E. Gourgoulhon, An introduction to relativistic hydrodynamics, EAS Publ. Ser. 21 (2006) 43 [gr-qc/0603009] [INSPIRE]. 